\title{
Probabilistic interpretation for systems of Isaacs equations with two reflecting barriers
}

\author{
Rainer Buckdahn and Juan Li
}

\begin{abstract}
In this paper we investigate zero-sum two-player stochastic differential games whose cost functionals are given by doubly controlled reflected backward stochastic differential equations (RBSDEs) with two barriers. For admissible controls which can depend on the whole past and so include, in particular, information occurring before the beginning of the game, the games are interpreted as games of the type "admissible strategy" against "admissible control", and the associated lower and upper value functions are studied. A priori random, they are shown to be deterministic, and it is proved that they are the unique viscosity solutions of the associated upper and the lower Bellman-Isaacs equations with two barriers, respectively. For the proofs we make full use of the penalization method for RBSDEs with one barrier and RBSDEs with two barriers. For this end we also prove new estimates for RBSDEs with two barriers, which are sharper than those in Hamadène, Hassani (Probab Theory Relat Fields 132:237-264, 2005). Furthermore, we show that the viscosity solution of the Isaacs equation with two reflecting barriers not only can be approximated by the viscosity solutions of penalized Isaacs equations with one barrier, but also directly by the viscosity solutions of penalized Isaacs equations without barrier.
\end{abstract}

Mathematics Subject Classification (2000). 93E05, 90C39, 60H10.

Keywords. Stochastic differential games, Zero-sum games, Value function, Reflected backward stochastic differential equations, Dynamic programming principle, Isaacs equations with obstacles, Viscosity solution.

\section{Introduction}

In this paper we study two-player zero-sum stochastic differential games whose cost functionals are given by doubly controlled backward stochastic differential equations (RBSDEs) with two reflecting barriers. Fleming and Souganidis [12] 
were the first to study in a rigorous manner two-player zero-sum stochastic differential games. They namely proved that the lower and the upper value functions of such games satisfy the dynamic programming principle, and that they are the unique viscosity solutions of the associated Bellman-Isaacs equations and coincide under the Isaacs condition. So a lot of recent works are based on the ideas developed in their pioneering paper [12]; see, for instance, [2-4,19]. The reader interested in this subject is also referred to the references given in [12].

On the other hand, general non-linear backward stochastic differential equations (BSDEs) were first introduced by Pardoux and Peng [24] in 1990. They have been studied since then by a lot of authors and have found various applications, namely in stochastic control, finance and the second order PDE theory (for more details, see, for instance [9] and the references therein). BSDE methods, originally developed by Peng $[25,26]$ for the stochastic control theory, have been introduced in the theory of stochastic differential games by Hamadène, Lepeltier [14] and Hamadène et al. [17] for the study of games with a dynamics whose diffusion coefficient is strictly elliptic and doesn't depend on controls. In Buckdahn and Li [3] there isn't any such restriction on the diffusion coefficient and they used a completely new approach to study stochastic differential games. In their framework, in difference to that of [12], the admissible controls can depend on the whole past, including information occurring before the beginning of the game, and, with the help of a Girsanov transformation argument, the a priori random lower and upper value functions were shown to be deterministic. This new approach in combination with BSDE methods (in particular the notion of stochastic backward semigroups, see Peng [25]) allowed them to prove the dynamic programming principle (DPP) for the upper and lower value functions of the game as well as to study the associated Isaacs equations in a very straightforward way (i.e., in particular without making use of so called $r$-strategies and $\pi$-admissible strategies playing a crucial role in [12]).

Also in this paper we investigate two-player zero-sum stochastic differential games, but in difference to the setting chosen in the papers mentioned above, we consider now more general running cost functionals which are defined by doubly controlled RBSDEs with two barriers. Cvitanic and Karatzas [6] were the first to introduce RBSDEs with two reflecting barriers and to study the existence and the uniqueness for this type of equation. An RBSDE can be understood as a BSDE whose solution is forced to stay between two prescribed continuous processes $L$ and $U$, called the lower and the upper obstacle, respectively. The forces imposing the reflection of the first component $Y$ of the solution of the BSDE at the lower and the upper barrier, respectively, are described by two continuous increasing processes $K^{+}$and $K^{-}$; they are a part of the solution quadruplet of the RBSDE. Cvitanic and Karatzas also established the connection between RBSDEs and Dynkin games. Their work has generalized an earlier one by El Karoui et al. [10] studying BSDEs reflected at a single barrier. Since then, motivated by the various applications of the RBSDEs, namely those to stochastic differential games as well as in finance, many authors have worked on this subject. We refer, 
for instance, to the papers $[11,13-18,23,27]$ but also to the references in these papers. In [6] the authors used two different approaches for their existence and uniqueness proof, one is based on Picard's method and Dynkin games, the other on the penalization method. Both approaches led to different types of conditions on the barriers: while one approach used the Mokobodzki condition, the other one supposed the regularity in the sense that the barriers are uniformly approximated by Itô processes. Hamadène and Hassani [18] replaced these conditions, which are rather difficult to verify in concrete applications, by the condition that the both barriers $U$ and $L$ are completely separated, i.e., $L_{t}<U_{t}$, a.s., for all time points $t$. In our paper we adopt this condition on the barriers.

The cost functionals defined by the doubly controlled RBSDEs with two barriers are described the payoff for Player I and the cost for Player II; they are, in particular, random variables. As well known for stochastic differential games, the players cannot restrict to play only control processes; one player has to fix a strategy while the other player chooses the best answer to this strategy in form of a control process. So the lower value function $W$ is defined as the essential infimum of the essential supremum of all cost functionals, where the essential infimum is taken over all admissible strategies of Player II and the essential supremum is taken over all admissible controls of Player I. The upper value function $U$ is defined by changing the roles of the both players: as the essential supremum of the essential infimum of all cost functionals, where the essential supremum is taken over all admissible strategies of Player I and the essential infimum is taken over all admissible controls of Player II; for the precise definitions see (3.9) and (3.10). The objective of our paper is to investigate these lower and upper value functions $W$ and $U$. The main results of the paper state that $W$ and $U$ are deterministic (Proposition 3.1) continuous viscosity solutions of associated Bellman-Isaacs equations with two obstacles (Theorem 4.1), and they satisfy the DPP (Theorem $3.1)$.

We emphasize that the random fields $W$ and $U$, introduced as combination of essential infimum and essential supremum over a class of essentially bounded random variables, are deterministic is far from being trivial. For the proof of Proposition 3.1 we adapt the argument developed by Buckdahn and Li [3]. Proposition 3.1 then allows to prove the DPP in a straightforward way with the help of the method of stochastic backward semigroups introduced by Peng [25], which is here extended to RBSDEs with two barriers. Another key element in the proof of the DPP is the improvement of former estimates for BSDE with two barriers, obtained by [18]. In fact, we prove that, in the Markovian framework, under appropriate assumptions the dependence of the solution on the random initial value of the driving SDE (on which also the barrier processes depend) is Lipschitz continuous (Proposition 6.1).

The proof that the lower and upper value functions are viscosity solutions of the associated Isaacs equations with obstacles (Theorem 4.1) is based on a penalization method. As a byproduct we obtain that the viscosity solutions of penalized Isaacs equations with a lower barrier as well as of those with an upper barrier 
(see (4.7), (4.8)) converge to the viscosity solution of the Isaacs equation with two obstacles (4.1) (Theorem 4.2). We also obtain that the viscosity solution of penalized Isaacs equations without obstacles (see (4.14)) converges to the viscosity solution of Eq. (4.1) (Theorem 4.3). Moreover, similar to Buckdahn and Li [3,4] we prove the uniqueness (Theorem 5.1) in a class of continuous functions with a growth condition which was introduced by Barles, Buckdahn and Pardoux [1] and is weaker than the polynomial growth assumption.

Our paper is organized as follows. Sections 2 recalls some elements of the theory of BSDES and RBSDEs with one barrier and two barriers, respectively, which will be needed in the sequel. Section 3 introduces the setting of stochastic differential games with two reflecting barriers and their lower and upper value functions $W$ and $U$. It is proved there that these both functions are deterministic (Proposition 3.1) and satisfy the DPP (Theorem 3.1). In Sect. 4 we show that $W$ and $U$ are viscosity solutions of the associated Bellman-Isaacs equations with two barriers (Theorem 4.1); their uniqueness is studied in Sect. 5. We also study approximations of $W$ and $U$ by viscosity solutions of Isaacs equations with one or even without obstacles (Theorems 4.2 and 4.3). Finally, after having characterized $W$ and $U$ as the unique viscosity solutions of the associated Bellman-Isaacs equations with two barriers we show that $W$ is less than or equal to $U$, and that, under the Isaacs condition, $W$ and $U$ coincide (one says that the game has a value). For the sake of readability of the paper the presentation of some basic properties of RBSDEs with two barriers associated with forward SDEs, which are needed for our studies, is postponed to the appendix (Sect. 6). However, it is not only a recall, some new results on RBSDEs with two barriers are also given there, namely Proposition 6.1, already mentioned above. On the other hand, the proof of DPP (Theorem 3.1) is also given in the appendix.

\section{Preliminaries}

The purpose of this section is to introduce some basic notions and results concerning BSDEs and RBSDEs with one and two barriers, which will be needed in the subsequent sections. In all that follows we will work on the classical Wiener space $(\Omega, \mathcal{F}, P)$ : For an arbitrarily fixed time horizon $T>0, \Omega$ is the set of all continuous functions from $[0, T]$ to $\mathbb{R}^{d}$, with initial value $0\left(\Omega=C_{0}\left([0, T] ; \mathbb{R}^{d}\right)\right)$ and $\mathcal{F}$ is the Borel $\sigma$-algebra over $\Omega$, completed by the Wiener measure on $P$. On this probability space the coordinate process $B_{s}(\omega)=\omega_{s}, s \in[0, T], \omega \in \Omega$, is a $d$-dimensional Brownian motion. By $\mathbb{F}=\left\{\mathcal{F}_{s}, 0 \leq s \leq T\right\}$ we denote the natural filtration generated by the coordinate process $B$ and augmented by all P-null sets, i.e.,

$$
\mathcal{F}_{s}=\sigma\left\{B_{r}, r \leq s\right\} \vee \mathcal{N}_{P}, \quad s \in[0, T]
$$

Here $\mathcal{N}_{P}$ is the set of all P-null sets. 
We also shall introduce the following both spaces of processes which will be used frequently in the sequel:

$\mathcal{S}^{2}(0, T ; \mathbb{R})$ is the set of real-valued adapted continuous process $\left(\psi_{t}\right)_{0 \leq t \leq T}$ such that $E\left[\sup _{0 \leq t \leq T}\left|\psi_{t}\right|^{2}\right]<+\infty ;$

$\cdot \mathcal{H}^{2}\left(0, T ; \mathbb{R}^{n}\right)\left(\right.$ resp., $\left.\mathcal{H}\left(0, T ; \mathbb{R}^{n}\right)\right)$ is the set of $\mathbb{R}^{n}$-valued progressively measurable process $\left(\psi_{t}\right)_{0 \leq t \leq T}$ such that $\|\psi\|^{2}=E\left[\int_{0}^{T}\left|\psi_{t}\right|^{2} d t\right]<+\infty$ (resp., $\int_{0}^{T}\left|\psi_{t}\right|^{2} d t<+\infty$, P-a.s.);

$\cdot \mathcal{A}_{\mathrm{C}}(0, T ; \mathbb{R})\left(\operatorname{resp} ., \mathcal{A}_{\mathrm{C}}^{2}(0, T ; \mathbb{R})\right)$ is the set of real-valued adapted continuous non-decreasing process $\left(\psi_{t}\right)_{0 \leq t \leq T}$ such that $\psi_{0}=0$ (resp., if, additionally, $E\left[\left|\psi_{T}\right|^{\overline{2}}\right]<+\infty$ );

(recall that $|z|$ denotes the Euclidean norm of $z \in \mathbb{R}^{n}$ ). Let us now consider a measurable function $g: \Omega \times[0, T] \times \mathbb{R} \times \mathbb{R}^{d} \rightarrow \mathbb{R}$ with the property that $(g(t, y, z))_{t \in[0, T]}$ is $\mathbb{F}$-progressively measurable for all $(y, z)$ in $\mathbb{R} \times \mathbb{R}^{d}$. We make the following standard assumptions on $g$ throughout the paper:

(A1) There is some real $C \geq 0$ such that, P-a.s., for all $t \in[0, T], y_{1}, y_{2} \in$ $\mathbb{R}, z_{1}, z_{2} \in \mathbb{R}^{d},\left|g\left(t, y_{1}, z_{1}\right)-g\left(t, y_{2}, z_{2}\right)\right| \leq C\left(\left|y_{1}-y_{2}\right|+\left|z_{1}-z_{2}\right|\right)$.

(A2) $g(\cdot, 0,0) \in \mathcal{H}^{2}(0, T ; \mathbb{R})$.

The following result on BSDEs is by now well known; for its proof the reader is referred, for instance to the pioneering work by Pardoux and Peng [24], but also to $[9]$.

Lemma 2.1. Let the function $g$ satisfy the assumptions (A1) and (A2). Then, for any random variable $\xi \in L^{2}\left(\Omega, \mathcal{F}_{T}, P\right)$, the BSDE associated with $(g, \xi)$

$$
Y_{t}=\xi+\int_{t}^{T} g\left(s, Y_{s}, Z_{s}\right) d s-\int_{t}^{T} Z_{s} d B_{s}, \quad 0 \leq t \leq T
$$

has a unique adapted solution

$$
\left(Y_{t}, Z_{t}\right)_{t \in[0, T]} \in \mathcal{S}^{2}(0, T ; \mathbb{R}) \times \mathcal{H}^{2}\left(0, T ; \mathbb{R}^{d}\right) .
$$

Besides the existence and uniqueness result we shall also recall the comparison theorem for BSDEs (see Theorem 2.2 in El Karoui et al. [9] or Proposition 2.4 in Peng [26]).

Lemma 2.2. (Comparison Theorem) Given two coefficients $g_{1}$ and $g_{2}$ satisfying (A1) and (A2) and two terminal values $\xi_{1}, \xi_{2} \in L^{2}\left(\Omega, \mathcal{F}_{T}, P\right)$, we denote by $\left(Y^{1}, Z^{1}\right)$ and $\left(Y^{2}, Z^{2}\right)$ the solution of the BSDE with the data $\left(\xi_{1}, g_{1}\right)$ and $\left(\xi_{2}, g_{2}\right)$, respectively. Then we have:

(i) (Monotonicity) If $\xi_{1} \geq \xi_{2}$ and $g_{1} \geq g_{2}$, a.s., then $Y_{t}^{1} \geq Y_{t}^{2}$, for all $t \in[0, T]$, a.s.

(ii) (Strict Monotonicity) If, in addition to (i), we also assume that $P\left\{\xi_{1}>\xi_{2}\right\}>$ 0 , then $P\left\{Y_{t}^{1}>Y_{t}^{2}\right\}>0$, for all $0 \leq t \leq T$, and in particular, $Y_{0}^{1}>Y_{0}^{2}$. 


\subsection{Reflected BSDEs with one barrier}

After this short and very basic recall on BSDEs let us consider now RBSDEs with one barrier. An RBSDE with one barrier is associated with a terminal condition $\xi \in L^{2}\left(\Omega, \mathcal{F}_{T}, P\right)$, a generator $g$ and an "obstacle" process $\left\{L_{t}\right\}_{0 \leq t \leq T}$. We assume that $\left\{L_{t}\right\}_{0 \leq t \leq T} \in \mathcal{S}^{2}(0, T ; \mathbb{R})$ and $L_{T} \leq \xi$, a.s.

A solution of an RBSDE with one barrier is a triplet $(Y, Z, K)$ of $\mathbb{F}$-progressively measurable processes, taking its values in $\mathbb{R} \times \mathbb{R}^{d} \times \mathbb{R}_{+}$and satisfying the following properties

(i) $Y \in \mathcal{S}^{2}(0, T ; \mathbb{R}), Z \in \mathcal{H}^{2}\left(0, T ; \mathbb{R}^{d}\right)$ and $K \in \mathcal{A}_{c}^{2}(0, T ; \mathbb{R})$;

(ii) $Y_{t}=\xi+\int_{t}^{T} g\left(s, Y_{s}, Z_{s}\right) d s+K_{T}-K_{t}-\int_{t}^{T} Z_{s} d B_{s}, \quad t \in[0, T]$;

(iii) $Y_{t} \geq L_{t}$, a.s., for any $t \in[0, T]$;

(iv) $\int_{0}^{T}\left(Y_{t}-L_{t}\right) d K_{t}=0$.

The following two lemmata are borrowed from Theorems 5.2 and 4.1, respectively, of the paper [10].

Lemma 2.3. Assume that g satisfies (A1) and (A2), $\xi \in L^{2}\left(\Omega, \mathcal{F}_{T}, P\right),\left\{L_{t}\right\}_{0 \leq t \leq T} \in$ $\mathcal{S}^{2}(0, T ; \mathbb{R})$, and $L_{T} \leq \xi$ a.s. Then $R B S D E(2.2)$ has a unique solution $(Y, Z, K)$.

Remark 2.1. For shortness, a given triplet $(\xi, g, L)$ is said to satisfy the Standard Assumptions if the generator $g$ satisfies (A1) and (A2), the terminal value $\xi$ belongs to $L^{2}\left(\Omega, \mathcal{F}_{T}, P\right)$, and the obstacle process $L \in \mathcal{S}^{2}(0, T ; \mathbb{R})$ is such that $L_{T} \leq \xi$, a.s.

Lemma 2.4. (Comparison Theorem) We suppose that two triplets $\left(\xi_{1}, g_{1}, L^{1}\right)$ and $\left(\xi_{2}, g_{2}, L^{2}\right)$ satisfy the Standard Assumptions but assume only for one of the both coefficients $g_{1}$ and $g_{2}$ to fulfill the Lipschitz condition (A.1). Furthermore, we make the following assumptions:

(i) $\xi_{1} \leq \xi_{2}, \quad$ a.s.;

(ii) $g_{1}(t, y, z) \leq g_{2}(t, y, z)$, a.s., for $(t, y, z) \in[0, T] \times \mathbb{R} \times \mathbb{R}^{d}$;

(iii) $L_{t}^{1} \leq L_{t}^{2}$, a.s., for $t \in[0, T]$.

Let $\left(Y^{1}, Z^{1}, K^{1}\right)$ and $\left(Y^{2}, Z^{2}, K^{2}\right)$ be adapted solutions of RBSDEs (2.2) with data $\left(\xi_{1}, g_{1}, L^{1}\right)$ and $\left(\xi_{2}, g_{2}, L^{2}\right)$, respectively. Then, $Y_{t}^{1} \leq Y_{t}^{2}$, for all $t \in[0, T]$, a.s.

We will also need the following standard result on RBSDEs with one barrier.

Lemma 2.5. Let $(Y, Z, K)$ be the solution of the above RBSDE (2.2) with data $(\xi, g, L)$ satisfying the Standard Assumptions. Then, there exists a constant $C$ 
such that

$$
\begin{aligned}
E & {\left[\sup _{t \leq s \leq T}\left|Y_{s}\right|^{2}+\int_{t}^{T}\left|Z_{s}\right|^{2} d s+\left|K_{T}-K_{t}\right|^{2} \mid \mathcal{F}_{t}\right] \leq C E\left[\xi^{2}+\left(\int_{t}^{T} g(s, 0,0) d s\right)^{2}\right.} \\
& \left.+\sup _{t \leq s \leq T} L_{s}^{2} \mid \mathcal{F}_{t}\right] .
\end{aligned}
$$

The constant $C$ depends only on the Lipschitz constant of $g$.

Lemma 2.5 is based on Propositions 3.5 in [10].

\subsection{Reflected BSDEs with two barriers}

Let us consider now RBSDEs with two barriers. An RBSDE with two barriers is associated with a terminal condition $\xi \in L^{2}\left(\Omega, \mathcal{F}_{T}, P\right)$, a generator $g$ and two barriers $L:=\left\{L_{t}\right\}_{0 \leq t \leq T}$ and $U:=\left\{U_{t}\right\}_{0 \leq t \leq T}$ which belong to $\mathcal{S}^{2}(0, T ; \mathbb{R})$ and satisfy $L_{t}<U_{t}, 0 \leq t \leq T$, a.s., and $L_{T} \leq \xi \leq U_{T}$, a.s.

A solution of an RBSDE with two barriers is a quadruplet $\left(Y, Z, K^{+}, K^{-}\right)$ of $\mathbb{F}$-progressively measurable processes, taking its values in $\mathbb{R} \times \mathbb{R}^{d} \times \mathbb{R}_{+} \times \mathbb{R}_{+}$ and satisfying the following properties

(i) $Y \in \mathcal{S}^{2}(0, T ; \mathbb{R}), Z \in \mathcal{H}\left(0, T ; \mathbb{R}^{d}\right)$ and $K^{+}, K^{-} \in \mathcal{A}_{c}(0, T ; \mathbb{R})$;

(ii) $Y_{t}=\xi+\int_{t}^{T} g\left(s, Y_{s}, Z_{s}\right) d s+\left(K_{T}^{+}-K_{t}^{+}\right)-\left(K_{T}^{-}-K_{t}^{-}\right)$

$$
-\int_{t}^{T} Z_{s} d B_{s}, t \in[0, T]
$$

(iii) $L_{t} \leq Y_{t} \leq U_{t}$, a.s., for any $t \in[0, T]$;

(iv) $\int_{0}^{T}\left(Y_{t}-L_{t}\right) d K_{t}^{+}=\int_{0}^{T}\left(U_{t}-Y_{t}\right) d K_{t}^{-}=0$.

The following two lemmata are borrowed from Theorems 3.7 and 1.3 in Hamadène and Hassani [18], respectively.

Lemma 2.6. Assume that $g$ satisfies $(A 1)$ and $(A 2), \xi$ belongs to $L^{2}\left(\Omega, \mathcal{F}_{T}, P\right)$, and $L, U \in \mathcal{S}^{2}(0, T ; \mathbb{R})$ are such that $L_{t}<U_{t}, 0 \leq t \leq T$, a.s., and $L_{T} \leq \xi \leq U_{T}$, a.s. Then RBSDE (2.3) has a unique solution $\left(Y, Z, K^{+}, K^{-}\right)$.

Remark 2.2. For shortness, a given quadruplet $(\xi, g, L, U)$ is said to satisfy the Standard Assumptions if the generator $g$ fulfills (A1) and (A2), the terminal value $\xi$ belongs to $L^{2}\left(\Omega, \mathcal{F}_{T}, P\right)$, and the two barriers $L, U$ belong to $\mathcal{S}^{2}(0, T ; \mathbb{R})$ and satisfy $L_{t}<U_{t}, 0 \leq t \leq T$, a.s., and $L_{T} \leq \xi \leq U_{T}$, a.s.

Lemma 2.7. (Comparison Theorem) We suppose that the both quadruplets $\left(\xi_{1}, g_{1}\right.$, $\left.L^{1}, U^{1}\right)$ and $\left(\xi_{2}, g_{2}, L^{2}, U^{2}\right)$ satisfy the Standard Assumptions. Let $\left(Y^{1}, Z^{1}, K^{1+}\right.$, $\left.K^{1-}\right)$ and $\left(Y^{2}, Z^{2}, K^{2+}, K^{2-}\right)$ be adapted solutions of $R B S D E$ (2.3) with data 
$\left(\xi_{1}, g_{1}, L^{1}, U^{1}\right)$ and $\left(\xi_{2}, g_{2}, L^{2}, U^{2}\right)$, respectively. Furthermore, we make the following assumptions:

(i) $\xi_{1} \leq \xi_{2}$, a.s.;

(ii) $g_{1}\left(t, Y_{t}^{2}, Z_{t}^{2}\right) \leq g_{2}\left(t, Y_{t}^{2}, Z_{t}^{2}\right)$, a.s., for $t \in[0, T]$;

(iii) $L_{t}^{1} \leq L_{t}^{2}, U_{t}^{1} \leq U_{t}^{2}$, for all $t \in[0, T]$, a.s.

Then, $Y_{t}^{1} \leq Y_{t}^{2}$, for all $t \in[0, T]$, a.s. In addition if:

(i) $g_{1}(t, y, z) \leq g_{2}(t, y, z)$, a.s., for $(t, y, z) \in[0, T] \times \mathbb{R} \times \mathbb{R}^{d}$;

(ii) $L_{t}^{1}=L_{t}^{2}, U_{t}^{1}=U_{t}^{2}$, a.s., for $t \in[0, T]$,

then we also have: $K_{t}^{1-} \leq K_{t}^{2-}$, and $K_{t}^{1+} \geq K_{t}^{2+}$, for all $t \in[0, T]$, a.s.

We will also need the following two estimates for RBSDEs with two barriers.

Lemma 2.8. Let $\left(Y, Z, K^{+}, K^{-}\right)$be the solution of the above RBSDE (2.3) with data $(\xi, g, L, U)$ satisfying the Standard Assumptions. Then,

$$
E\left[\sup _{t \leq s \leq T}\left|Y_{s}\right|^{2} \mid \mathcal{F}_{t}\right] \leq E\left[\sup _{t \leq s \leq T}\left(\left|L_{s}\right|^{2} \vee\left|U_{s}\right|^{2}\right) \mid \mathcal{F}_{t}\right], \quad t \in[0, T] .
$$

Proof. Since $\left(Y, Z, K^{+}, K^{-}\right)$is the solution of Eq. (2.3), we have that $L_{t} \leq Y_{t} \leq$ $U_{t}$, a.s., the proof is obvious.

Lemma 2.9. Let $(\xi, g, L, U)$ and $\left(\xi^{\prime}, g^{\prime}, L, U\right)$ be two quadruplets satisfying the above Standard Assumptions. We suppose that $\left(Y, Z, K^{+}, K^{-}\right)$and $\left(Y^{\prime}, Z^{\prime}, K^{\prime}\right.$, $\left.K^{\prime}-\right)$ are the solutions of $R B S D E(2.3)$ with the data $(\xi, g, L, U)$ and $\left(\xi^{\prime}, g^{\prime}, L, U\right)$, respectively. Then there exists a constant $C$ such that, with the notation,

$$
\Delta \xi=\xi-\xi^{\prime}, \quad \Delta g=g-g^{\prime}, \quad \Delta Y=Y-Y^{\prime}, \quad \Delta Z=Z-Z^{\prime},
$$

it holds

$$
E\left[\sup _{t \leq s \leq T}\left|\Delta Y_{s}\right|^{2} \mid \mathcal{F}_{t}\right] \leq C E\left[|\Delta \xi|^{2}+\int_{t}^{T}\left|\Delta g\left(s, Y_{s}, Z_{s}\right)\right|^{2} d s \mid \mathcal{F}_{t}\right]
$$

The constant $C$ depends only on the Lipschitz constant of $g^{\prime}$ and $g$.

Proof. From Proposition 4.1 in Hamadène and Hassani [18] there exists a localizing increasing sequence of stopping times $\gamma_{n}: \Omega \rightarrow[0, T]$ such that:

(i) $P\left(\cup_{n \geq 1}\left\{\gamma_{n}=T\right\}\right)=1$;

(ii) $\forall n \geq 1: E\left[\left(K_{\gamma_{n}}^{+}\right)^{2}+\left(K_{\gamma_{n}}^{-}\right)^{2}+\int_{0}^{\gamma_{n}}\left|Z_{s}\right|^{2} d s\right]<\infty$;

$$
E\left[\left(K_{\gamma_{n}}^{\prime+}\right)^{2}+\left(K_{\gamma_{n}}^{-}\right)^{2}+\int_{0}^{\gamma_{n}}\left|Z_{s}^{\prime}\right|^{2} d s\right]<\infty .
$$

We remark that $\left(\gamma_{n}\right)_{n \geq 1}$ only depends on $L, U,(g(t, \omega, 0,0))_{t \leq T},\left(g^{\prime}(t, \omega, 0,0)\right)_{t \leq T}$, and Lipschitz constants of $g$ and $g^{\prime}$. 
Applying Itô's formula to $e^{\vartheta s}\left|Y_{s}-Y_{s}^{\prime}\right|^{2}$ we get

$$
\begin{aligned}
& e^{\vartheta\left(t \wedge \gamma_{n}\right)}\left|Y_{t \wedge \gamma_{n}}-Y_{t \wedge \gamma_{n}}^{\prime}\right|^{2}+\int_{t \wedge \gamma_{n}}^{\gamma_{n}} e^{\vartheta s}\left(\vartheta\left|Y_{s}-Y_{s}^{\prime}\right|^{2}+\left|Z_{s}-Z_{s}^{\prime}\right|^{2}\right) d s \\
& =e^{\vartheta \gamma_{n}}\left|Y_{\gamma_{n}}-Y_{\gamma_{n}}^{\prime}\right|^{2}+2 \int_{t \wedge \gamma_{n}}^{\gamma_{n}} e^{\vartheta s}\left(Y_{s}-Y_{s}^{\prime}\right)\left(g\left(s, Y_{s}, Z_{s}\right)-g^{\prime}\left(s, Y_{s}^{\prime}, Z_{s}^{\prime}\right)\right) d s \\
& \quad+2 \int_{t \wedge \gamma_{n}}^{\gamma_{n}} e^{\vartheta s}\left(Y_{s}-Y_{s}^{\prime}\right)\left(d K_{s}^{+}-d K_{s}^{\prime}\right)-2 \int_{t \wedge \gamma_{n}}^{\gamma_{n}} e^{\vartheta s}\left(Y_{s}-Y_{s}^{\prime}\right)\left(d K_{s}^{-}-d K_{s}^{\prime}\right) \\
& \quad-2 \int_{t \wedge \gamma_{n}}^{\gamma_{n}} e^{\vartheta s}\left(Y_{s}-Y_{s}^{\prime}\right)\left(Z_{s}-Z_{s}^{\prime}\right) d B_{s} .
\end{aligned}
$$

Notice that

$$
\begin{aligned}
& \int_{t \wedge \gamma_{n}}^{\gamma_{n}} e^{\vartheta s}\left(Y_{s}-Y_{s}^{\prime}\right)\left(d K_{s}^{+}-d K_{s}^{\prime}\right) \\
& =\int_{t \wedge \gamma_{n}}^{\gamma_{n}} e^{\vartheta s}\left(\left(Y_{s}-L_{s}\right)-\left(Y_{s}^{\prime}-L_{s}\right)\right)\left(d K_{s}^{+}-d K_{s}^{\prime}{ }^{+}\right) \\
& =-\int_{t \wedge \gamma_{n}}^{\gamma_{n}} e^{\vartheta s}\left(Y_{s}^{\prime}-L_{s}\right) d K_{s}^{+}-\int_{t \wedge \gamma_{n}}^{\gamma_{n}} e^{\vartheta s}\left(Y_{s}-L_{s}\right) d K_{s}^{\prime} \leq 0 .
\end{aligned}
$$

Similarly we have

$$
-\int_{t \wedge \gamma_{n}}^{\gamma_{n}} e^{\vartheta s}\left(Y_{s}-Y_{s}^{\prime}\right)\left(d K_{s}^{-}-d K_{s}^{\prime}{ }^{-}\right) \leq 0 .
$$

Since $E\left[\int_{0}^{\gamma_{n}}\left|Z_{s}-Z_{s}^{\prime}\right|^{2} d s\right]<\infty$, and $\gamma_{n}$ is a bounded stopping time we have that $\int_{0}^{\gamma_{n} \wedge} e^{\vartheta s}\left(Y_{s}-Y_{s}^{\prime}\right)\left(Z_{s}-Z_{s}^{\prime}\right) d B_{s}$ is a martingale. Hence, we can get

$$
\begin{aligned}
& E\left[e^{\vartheta\left(t \wedge \gamma_{n}\right)}\left|Y_{t \wedge \gamma_{n}}-Y_{t \wedge \gamma_{n}}^{\prime}\right|^{2}+\int_{t \wedge \gamma_{n}}^{\gamma_{n}} e^{\vartheta s}\left(\vartheta\left|Y_{s}-Y_{s}^{\prime}\right|^{2}+\left|Z_{s}-Z_{s}^{\prime}\right|^{2}\right) d s \mid \mathcal{F}_{t}\right] \\
& \leq E\left[e^{\vartheta \gamma_{n}}\left|Y_{\gamma_{n}}-Y_{\gamma_{n}}^{\prime}\right|^{2}+2 \int_{t \wedge \gamma_{n}}^{\gamma_{n}} e^{\vartheta s}\left(Y_{s}-Y_{s}^{\prime}\right)\left(g\left(s, Y_{s}, Z_{s}\right)-g^{\prime}\left(s, Y_{s}^{\prime}, Z_{s}^{\prime}\right)\right) d s \mid \mathcal{F}_{t}\right] \\
& \leq E\left[e^{\vartheta \gamma_{n}}\left|Y_{\gamma_{n}}-Y_{\gamma_{n}}^{\prime}\right|^{2}+\int_{t \wedge \gamma_{n}}^{\gamma_{n}} e^{\vartheta s}\left|\Delta g\left(s, Y_{s}, Z_{s}\right)\right|^{2} d s\right. \\
& \quad+2\left(L+L^{2}+1\right) \int_{t \wedge \gamma_{n}}^{\gamma_{n}} e^{\vartheta s}\left|Y_{s}-Y_{s}^{\prime}\right|^{2} d s \\
& \left.\quad+\frac{1}{2} \int_{t \wedge \gamma_{n}}^{\gamma_{n}} e^{\vartheta s}\left|Z_{s}-Z_{s}^{\prime}\right|^{2} d s \mid \mathcal{F}_{t}\right]
\end{aligned}
$$


where $L$ is the Lipschitz constant of $g$ and $g^{\prime}$. Consequently, for $\vartheta=4\left(L+L^{2}+\right.$ 1) +1 , we have

$$
\begin{aligned}
& \left|Y_{t \wedge \gamma_{n}}-Y_{t \wedge \gamma_{n}}^{\prime}\right|^{2}+E\left[\int_{t \wedge \gamma_{n}}^{\gamma_{n}}\left(\left|Y_{s}-Y_{s}^{\prime}\right|^{2}+\left|Z_{s}-Z_{s}^{\prime}\right|^{2}\right) d s \mid \mathcal{F}_{t}\right] \\
& \quad \leq C E\left[\left|Y_{\gamma_{n}}-Y_{\gamma_{n}}^{\prime}\right|^{2}+\int_{t \wedge \gamma_{n}}^{\gamma_{n}}\left|\Delta g\left(s, Y_{s}, Z_{s}\right)\right|^{2} d s \mid \mathcal{F}_{t}\right], t \in[0, T], n \geq 1
\end{aligned}
$$

where $C$ is independent of $n$. On the other hand, from the equations of $Y$ and $Y^{\prime}$ and the Burkholder-Davis-Gundy inequality we obtain

$$
\begin{aligned}
& E\left[\sup _{s \in\left[t, \gamma_{n}\right]}\left|Y_{s}-Y_{s}^{\prime}\right|^{2} \mid \mathcal{F}_{t}\right] \\
& \leq E\left[\left|Y_{\gamma_{n}}-Y_{\gamma_{n}}^{\prime}\right|^{2} \mid \mathcal{F}_{t}\right]+2 E\left[\int_{t \wedge \gamma_{n}}^{\gamma_{n}}\left|Y_{s}-Y_{s}^{\prime}\right|\left|g\left(s, Y_{s}, Z_{s}\right)-g\left(s, Y_{s}^{\prime}, Z_{s}^{\prime}\right)\right| d s \mid \mathcal{F}_{t}\right] \\
& +\frac{1}{2} E\left[\sup _{s \in\left[t, \gamma_{n}\right]}\left|Y_{s}-Y_{s}^{\prime}\right|^{2} \mid \mathcal{F}_{t}\right]+C E\left[\int_{t \wedge \gamma_{n}}^{\gamma_{n}}\left|Z_{s}-Z_{s}^{\prime}\right|^{2} d s \mid \mathcal{F}_{t}\right] .
\end{aligned}
$$

Then from the Lipschitz condition of $g$ and (2.4) we have, for some $C$ independent of $n$ :

$$
\begin{aligned}
& E\left[\sup _{s \in\left[t, \gamma_{n}\right]}\left|Y_{s}-Y_{s}^{\prime}\right|^{2} \mid \mathcal{F}_{t}\right] \\
& \quad \leq C\left(E\left[\left|Y_{\gamma_{n}}-Y_{\gamma_{n}}^{\prime}\right|^{2} \mid \mathcal{F}_{t}\right]+E\left[\int_{t \wedge \gamma_{n}}^{\gamma_{n}}\left|\Delta g\left(s, Y_{s}, Z_{s}\right)\right|^{2} d s \mid \mathcal{F}_{t}\right]\right), t \in[0, T] .
\end{aligned}
$$

Finally, the Lebesgue Dominated Convergence Theorem and the Monotonic Convergence Theorem yield

$$
\begin{aligned}
& E\left[\sup _{s \in[t, T]}\left|Y_{s}-Y_{s}^{\prime}\right|^{2} \mid \mathcal{F}_{t}\right] \\
& \quad \leq C\left(E\left[\left|\xi-\xi^{\prime}\right|^{2} \mid \mathcal{F}_{t}\right]+E\left[\int_{t}^{T}\left|\Delta g\left(s, Y_{s}, Z_{s}\right)\right|^{2} d s \mid \mathcal{F}_{t}\right]\right), \text { P-a.s., } t \in[0, T] .
\end{aligned}
$$

The proof is complete.

Remark 2.3. For the Markovian situation where the obstacle process is a deterministic function, we can improve Lemma 2.9 considerably and show that $Y$ is Lipschitz continuous with respect to the possibly random initial condition of the driving SDE (whose solution intervenes in the RBSDEs as well as in the obstacles), see Proposition 6.1 in the Sect. 6.

\section{Stochastic differential games with two barriers and associated dynamic programming principles}

We now introduce the framework for our study of stochastic differential games with reflection for two players. We denote the control state space of the first player by $U$, and that of the second one by $V$; the associated sets of admissible controls will be denoted by $\mathcal{U}$ and $\mathcal{V}$, respectively. The set $\mathcal{U}$ is formed by all $U$-valued $\mathbb{F}$-progressively measurable processes and $\mathcal{V}$ is the set of all $V$-valued $\mathbb{F}$-progressively measurable processes. The control state spaces $\mathrm{U}$ and $\mathrm{V}$ are supposed to be compact metric spaces. 
For given admissible controls $u(\cdot)=\left(u_{s}\right)_{s \in[0, T]} \in \mathcal{U}$ and $v(\cdot)=\left(v_{s}\right)_{s \in[0, T]} \in$ $\mathcal{V}$, the according orbit which regards $t$ as the initial time and $\zeta \in L^{2}\left(\Omega, \mathcal{F}_{t}, P ; \mathbb{R}^{n}\right)$ as the initial state is defined by the solution of the following SDE:

$$
\left\{\begin{array}{l}
d X_{s}^{t, \zeta ; u, v}=b\left(s, X_{s}^{t, \zeta ; u, v}, u_{s}, v_{s}\right) d s+\sigma\left(s, X_{s}^{t, \zeta ; u, v}, u_{s}, v_{s}\right) d B_{s}, s \in[t, T], \\
X_{t}^{t, \zeta ; u, v}=\zeta
\end{array}\right.
$$

where the mappings

$$
b:[0, T] \times \mathbb{R}^{n} \times U \times V \rightarrow \mathbb{R}^{n} \text { and } \sigma:[0, T] \times \mathbb{R}^{n} \times U \times V \rightarrow \mathbb{R}^{n \times d}
$$

satisfy the following conditions:

(i) For every fixed $x \in \mathbb{R}^{n}, b(., x, . .$.$) and \sigma(., x, .,$.$) are continuous in (t, u, v)$;

(ii) There exists a $C>0$ such that, for all $t \in[0, T], x, x^{\prime} \in \mathbb{R}^{n}, u \in U, v \in V$,

$$
\left|b(t, x, u, v)-b\left(t, x^{\prime}, u, v\right)\right|+\left|\sigma(t, x, u, v)-\sigma\left(t, x^{\prime}, u, v\right)\right| \leq C\left|x-x^{\prime}\right| .
$$

From (H3.1) we can get a global linear growth condition for $b$ and $\sigma$, i.e., the existence of some $C>0$ such that, for all $0 \leq t \leq T, u \in U, v \in V, x \in \mathbb{R}^{n}$,

$$
|b(t, x, u, v)|+|\sigma(t, x, u, v)| \leq C(1+|x|) .
$$

As recalled (6.2) in Sect. 6, it follows that, under the above assumptions, for any $u(\cdot) \in \mathcal{U}$ and $v(\cdot) \in \mathcal{V}, \operatorname{SDE}(3.1)$ has a unique strong solution. Moreover, for any $p \geq 2$, there exists $C_{p} \in \mathbb{R}$ such that, for any $t \in[0, T], u(\cdot) \in \mathcal{U}, v(\cdot) \in \mathcal{V}$ and $\zeta, \zeta^{\prime} \in L^{p}\left(\Omega, \mathcal{F}_{t}, P ; \mathbb{R}^{n}\right)$, we also have the following estimates, P-a.s.:

$$
\begin{aligned}
E\left[\sup _{s \in[t, T]}\left|X_{s}^{t, \zeta ; u, v}-X_{s}^{t, \zeta^{\prime} ; u, v}\right| p \mid \mathcal{F}_{t}\right] & \leq C_{p}\left|\zeta-\zeta^{\prime}\right|^{p}, \\
E\left[\sup _{s \in[t, T]}\left|X_{s}^{t, \zeta ; u, v}\right|^{p} \mid \mathcal{F}_{t}\right] & \leq C_{p}\left(1+|\zeta|^{p}\right) .
\end{aligned}
$$

The constant $C_{p}$ depends only on the Lipschitz and the linear growth constants of $b$ and $\sigma$ with respect to $x$. If it's obvious that $C$ depends on $p$ and doesn't provoke confusion in what follows we will omit the subscript $p$ for the simplicity of the notation.

Let now be given four functions

$$
\Phi: \mathbb{R}^{n} \rightarrow \mathbb{R}, h, h^{\prime}:[0, T] \times \mathbb{R}^{n} \rightarrow \mathbb{R}, f:[0, T] \times \mathbb{R}^{n} \times \mathbb{R} \times \mathbb{R}^{d} \times U \times V \rightarrow \mathbb{R}
$$

that satisfy the following conditions:

(i) For every fixed $(x, y, z) \in \mathbb{R}^{n} \times \mathbb{R} \times \mathbb{R}^{d}, f(., x, y, z, .,$.$) is continuous in (t, u, v)$ and there exists a constant $C>0$ such that, for all $t \in[0, T], x, x^{\prime} \in \mathbb{R}^{n}$, $y, y^{\prime} \in \mathbb{R}, z, z^{\prime} \in \mathbb{R}^{d}, u \in U$ and $v \in V$,

$$
\begin{aligned}
\mid f(t, x, y, z, u, v)- & f\left(t, x^{\prime}, y^{\prime}, z^{\prime}, u, v\right) \mid \\
\leq & C\left(\left|x-x^{\prime}\right|+\left|y-y^{\prime}\right|+\left|z-z^{\prime}\right|\right) ;
\end{aligned}
$$

(ii) There is a constant $C>0$ such that, for all $x, x^{\prime} \in \mathbb{R}^{n}$,

$$
\left|\Phi(x)-\Phi\left(x^{\prime}\right)\right| \leq C\left|x-x^{\prime}\right|
$$


(iii) For every fixed $x \in \mathbb{R}^{n}, h(., x), h^{\prime}(., x)$ are continuous in $t$ and there is a constant $C>0$ such that, for all $t \in[0, T], x, x^{\prime} \in \mathbb{R}^{n}$,

$$
\left|h(t, x)-h\left(t, x^{\prime}\right)\right|+\left|h^{\prime}(t, x)-h^{\prime}\left(t, x^{\prime}\right)\right| \leq C\left|x-x^{\prime}\right| .
$$

Moreover,

$$
h(t, x)<h^{\prime}(t, x), h(T, x) \leq \Phi(x) \leq h^{\prime}(T, x) \text {, for all } t \in[0, T], x \in \mathbb{R}^{n} .
$$

From (H3.2) we see that $f, h, h^{\prime}$ and $\Phi$ also satisfy the global linear growth condition in $x$, i.e., there exists some $C>0$ such that, for all $0 \leq t \leq T, u \in$ $U, v \in V, x \in \mathbb{R}^{n}$,

$$
|f(t, x, 0,0, u, v)|+|\Phi(x)|+|h(t, x)|+\left|h^{\prime}(t, x)\right| \leq C(1+|x|) .
$$

Let $t \in[0, T]$. For any $u(\cdot) \in \mathcal{U}, v(\cdot) \in \mathcal{V}$ and $\zeta \in L^{2}\left(\Omega, \mathcal{F}_{t}, P ; \mathbb{R}^{n}\right)$, the mappings $\xi:=\Phi\left(X_{T}^{t, \zeta ; u, v}\right), L_{s}=h\left(s, X_{s}^{t, \zeta ; u, v}\right), U_{s}=h^{\prime}\left(s, X_{s}^{t, \zeta ; u, v}\right)$ and $g(s, y, z):=$ $f\left(s, X_{s}^{t, \zeta ; u, v}, y, z, u_{s}, v_{s}\right)$ satisfy the conditions of Lemma 2.6 on the interval $[t, T]$. Therefore, there exists a unique solution to the following RBSDE with two barriers:

(i) $Y^{t, \zeta ; u, v} \in \mathcal{S}^{2}(t, T ; \mathbb{R}), Z^{t, \zeta ; u, v} \in \mathcal{H}\left(t, T ; \mathbb{R}^{d}\right)$, and $K^{+, t, \zeta ; u, v}$,

$$
K^{-, t, \zeta ; u, v} \in \mathcal{A}_{c}(t, T ; \mathbb{R}) ;
$$

(ii) $Y_{s}^{t, \zeta ; u, v}=\Phi\left(X_{T}^{t, \zeta ; u, v}\right)+\int_{s}^{T} f\left(r, X_{r}^{t, \zeta ; u, v}, Y_{r}^{t, \zeta ; u, v}, Z_{r}^{t, \zeta ; u, v}, u_{r}, v_{r}\right) d r$

$$
\begin{aligned}
& +\left(K_{T}^{+, t, \zeta ; u, v}-K_{s}^{+, t, \zeta ; u, v}\right)-\left(K_{T}^{-, t, \zeta ; u, v}-K_{s}^{-, t, \zeta ; u, v}\right) \\
& -\int_{s}^{T} Z_{r}^{t, \zeta ; u, v} d B_{r}, \quad s \in[t, T] ;
\end{aligned}
$$

(iii) $h\left(s, X_{s}^{t, \zeta ; u, v}\right) \leq Y_{s}^{t, \zeta ; u, v} \leq h^{\prime}\left(s, X_{s}^{t, \zeta ; u, v}\right)$, a.s., for any $s \in[t, T]$;

(iv) $\int_{t}^{T}\left(Y_{r}^{t, \zeta ; u, v}-h\left(r, X_{r}^{t, \zeta ; u, v}\right)\right) d K_{r}^{+, t, \zeta ; u, v}=\int_{t}^{T}\left(h^{\prime}\left(r, X_{r}^{t, \zeta ; u, v}\right)\right.$

$$
\left.-Y_{r}^{t, \zeta ; u, v}\right) d K_{r}^{-, t, \zeta ; u, v}=0,
$$

where $X^{t, \zeta ; u, v}$ is introduced by Eq. (3.1).

Moreover, from Proposition 6.1, we can see that there exists some constant $C>0$ such that, for all $0 \leq t \leq T, \zeta, \zeta^{\prime} \in L^{2}\left(\Omega, \mathcal{F}_{t}, P ; \mathbb{R}^{n}\right), u(\cdot) \in \mathcal{U}$ and $v(\cdot) \in \mathcal{V}$, P-a.s.,

(i) $\left|Y_{t}^{t, \zeta ; u, v}-Y_{t}^{t, \zeta^{\prime} ; u, v}\right| \leq C\left|\zeta-\zeta^{\prime}\right|$;

(ii) $\left|Y_{t}^{t, \zeta ; u, v}\right| \leq C(1+|\zeta|)$.

Now, similar to [3,12], we introduce the following subspaces of admissible controls and the definition of admissible strategies for the game:

Defnition 3.1. An admissible control process $u=\left\{u_{r}, r \in[t, s]\right\}$ (resp., $v=$ $\left.\left\{v_{r}, r \in[t, s]\right\}\right)$ for Player I (resp., II) on $[t, s](t<s \leq T)$ is an $\mathbb{F}$-progressively 
measurable process taking values in $\mathrm{U}$ (resp., V). The set of all admissible controls for Player I (resp., II) on $[t, s]$ is denoted by $\mathcal{U}_{t, s}\left(\right.$ resp., $\left.\mathcal{V}_{t, s}\right)$. We identify two processes $u$ and $\bar{u}$ in $\mathcal{U}_{t, s}$ and write $u \equiv \bar{u}$ on $[t, s]$, if $P\{u=\bar{u}$ a.e. in $[t, s]\}=1$. Similarly, we interpret $v \equiv \bar{v}$ on $[t, s]$ for two elements $v$ and $\bar{v}$ of $\mathcal{V}_{t, s}$.

Defnition 3.2. A nonanticipative strategy for Player I on $[t, s](t<s \leq T)$ is a mapping $\alpha: \mathcal{V}_{t, s} \longrightarrow \mathcal{U}_{t, s}$ such that, for any $\mathbb{F}$-stopping time $S: \Omega \rightarrow[t, s]$ and any $v_{1}, v_{2} \in \mathcal{V}_{t, s}$ with $v_{1} \equiv v_{2}$ on $\llbracket t, S \rrbracket$, it holds $\alpha\left(v_{1}\right) \equiv \alpha\left(v_{2}\right)$ on $\llbracket t, S \rrbracket$. Nonanticipative strategies for Player II on $[t, s], \beta: \mathcal{U}_{t, s} \longrightarrow \mathcal{V}_{t, s}$, are defined similarly. The set of all nonanticipative strategies $\alpha: \mathcal{V}_{t, s} \longrightarrow \mathcal{U}_{t, s}$ for Player I on $[t, s]$ is denoted by $\mathcal{A}_{t, s}$. The set of all nonanticipative strategies $\beta: \mathcal{U}_{t, s} \longrightarrow \mathcal{V}_{t, s}$ for Player II on $[t, s]$ is denoted by $\mathcal{B}_{t, s}$. (Recall that $\llbracket t, S \rrbracket=\{(r, \omega) \in[0, T] \times \Omega, t \leq r \leq S(\omega)\}$.)

For given control processes $u(\cdot) \in \mathcal{U}_{t, T}$ and $v(\cdot) \in \mathcal{V}_{t, T}$ we introduce the following associated cost functional

$$
J(t, x ; u, v):=Y_{t}^{t, x ; u, v},(t, x) \in[0, T] \times \mathbb{R}^{n},
$$

for which the process $Y^{t, x ; u, v}$ is defined by RBSDE (3.5).

From Proposition 6.2 (see: Appendix) we get that, for any $t \in[0, T], \zeta \in$ $L^{2}\left(\Omega, \mathcal{F}_{t}\right.$, $\left.P ; \mathbb{R}^{n}\right)$,

$$
J(t, \zeta ; u, v)=Y_{t}^{t, \zeta ; u, v}, \mathrm{P} \text {-a.s. }
$$

We emphasize that $J(t, \zeta ; u, v)=\left.J(t, x ; u, v)\right|_{x=\zeta}$ while $Y^{t, \zeta ; u, v}$ is defined by (3.5). Being particularly interested in the case of a deterministic $\zeta$, i.e., $\zeta=x \in \mathbb{R}^{n}$, we define the lower value function of our stochastic differential game with reflection

$$
W(t, x):=\operatorname{essinf}_{\beta \in \mathcal{B}_{t, T}} \operatorname{esssup}_{u \in \mathcal{U}_{t, T}} J(t, x ; u, \beta(u))
$$

and its upper value function

$$
U(t, x):=\operatorname{esssup}_{\alpha \in \mathcal{A}_{t, T}} \operatorname{essinf}_{v \in \mathcal{V}_{t, T}} J(t, x ; \alpha(v), v) .
$$

The names "lower value function" and "upper value function" for $W$ and $U$, respectively, are justified later by Remark 5.1 .

Remark 3.1. (1) Here the essential infimum and the essential supremum should be understood as one with respect to indexed families of random variables (see, e.g., Dunford and Schwartz [8], Dellacherie [7] or the appendix in Karatzas and Shreve [22] for detailed discussions). The reader is also referred to Remark 3.1 in $[3]$.

(2) Obviously, under the assumptions (H3.1-H3.2) it is a consequence of (3.6)-(ii) that the lower value function $W(t, x)$ as well as the upper value function $U(t, x)$ are well-defined and essentially bounded, $\mathcal{F}_{t}$-measurable random variables. But it turns out that $W(t, x)$ and $U(t, x)$ are even deterministic. For proving this we adapt the new approach by Buckdahn and $\mathrm{Li}[3,4]$. In the sequel we will concentrate on the study of the properties of $W$. The discussion of the properties of $U$, which are comparable with those of $W$, can be carried out in a similar manner. 
Proposition 3.1. For any $(t, x) \in[0, T] \times \mathbb{R}^{n}$, we have $W(t, x)=E[W(t, x)]$, $P$-a.s. Identifying $W(t, x)$ with its deterministic version $E[W(t, x)]$ we can consider $W:[0, T] \times \mathbb{R}^{n} \longrightarrow \mathbb{R}$ as a deterministic function.

Proof. We recall that $\Omega=C_{0}\left([0, T] ; \mathbb{R}^{d}\right)$ and denote by $H$ the Cameron-Martin space of all absolutely continuous elements $h \in \Omega$ whose derivative $\dot{h}$ belongs to $L^{2}\left([0, T], \mathbb{R}^{d}\right)$. For any $h \in H$, we define the mapping $\tau_{h} \omega:=\omega+h, \omega \in \Omega$. Obviously, $\tau_{h}: \Omega \rightarrow \Omega$ is a bijection and its law is given by $P \circ\left[\tau_{h}\right]^{-1}=$ $\exp \left\{\int_{0}^{T} \dot{h}_{s} d B_{s}-\frac{1}{2} \int_{0}^{T}\left|\dot{h}_{s}\right|^{2} d s\right\} P$. Let $(t, x) \in[0, T] \times \mathbb{R}^{n}$ be arbitrarily fixed and put $H_{t}=\{h \in H \mid h(\cdot)=h(\cdot \wedge t)\}$. We split now the proof in the following steps: $1^{\text {st }}$ step: For any $u \in \mathcal{U}_{t, T}, v \in \mathcal{V}_{t, T}, h \in H_{t}, J(t, x ; u, v)\left(\tau_{h}\right)=J\left(t, x ; u\left(\tau_{h}\right)\right.$, $\left.v\left(\tau_{h}\right)\right)$, P-a.s.

Indeed, for $h \in H_{t}$ we apply the Girsanov transformation to SDE (3.1) (with $\zeta=x)$. Notice that since $h \in H_{t}$, we have $d B_{s}\left(\tau_{h}\right)=d B_{s}, s \in[t, T]$. We compare the thus obtained equation with the SDE got from (3.1) by substituting the transformed control processes $u\left(\tau_{h}\right), v\left(\tau_{h}\right)$ for $u$ and $v$. Then, from the uniqueness of the solution of (3.1) we get $X_{s}^{t, x ; u, v}\left(\tau_{h}\right)=X_{s}^{t, x ; u\left(\tau_{h}\right), v\left(\tau_{h}\right)}$, for any $s \in[t, T]$, P-a.s. Furthermore, by a similar Girsanov transformation argument we get from the uniqueness of the solution of RBSDE (3.5),

$$
\begin{gathered}
Y_{s}^{t, x ; u, v}\left(\tau_{h}\right)=Y_{s}^{t, x ; u\left(\tau_{h}\right), v\left(\tau_{h}\right)}, \text { for any } s \in[t, T], \mathrm{P} \text {-a.s., } \\
Z_{s}^{t, x ; u, v}\left(\tau_{h}\right)=Z_{s}^{t, x ; u\left(\tau_{h}\right), v\left(\tau_{h}\right)}, \text { dsdP-a.e. on }[t, T] \times \Omega, \\
K_{s}^{+, t, x ; u, v}\left(\tau_{h}\right)=K_{s}^{+, t, x ; u\left(\tau_{h}\right), v\left(\tau_{h}\right)}, \text { for any } s \in[t, T], \mathrm{P} \text {-a.s., } \\
K_{s}^{-, t, x ; u, v}\left(\tau_{h}\right)=K_{s}^{-, t, x ; u\left(\tau_{h}\right), v\left(\tau_{h}\right)}, \text { for any } s \in[t, T], \mathrm{P} \text {-a.s. }
\end{gathered}
$$

This implies, in particular, that

$$
J(t, x ; u, v)\left(\tau_{h}\right)=J\left(t, x ; u\left(\tau_{h}\right), v\left(\tau_{h}\right)\right) \text {, P-a.s. }
$$

$2^{\text {nd }}$ step: For $\beta \in \mathcal{B}_{t, T}, h \in H_{t}$, let $\beta^{h}(u):=\beta\left(u\left(\tau_{-h}\right)\right)\left(\tau_{h}\right), u \in \mathcal{U}_{t, T}$. Then $\beta^{h} \in \mathcal{B}_{t, T}$.

Obviously, $\beta^{h}$ maps $\mathcal{U}_{t, T}$ into $\mathcal{V}_{t, T}$. Moreover, this mapping is nonanticipating. Indeed, let $S: \Omega \rightarrow[t, T]$ be an $\mathbb{F}$-stopping time and $u_{1}, u_{2} \in \mathcal{U}_{t, T}$ with $u_{1} \equiv u_{2}$ on $\llbracket t, S \rrbracket$. Then, obviously, $u_{1}\left(\tau_{-h}\right) \equiv u_{2}\left(\tau_{-h}\right)$ on $\llbracket t, S\left(\tau_{-h}\right) \rrbracket$ (notice that $S\left(\tau_{-h}\right)$ is still a stopping time), and because $\beta \in \mathcal{B}_{t, T}$ we have $\beta\left(u_{1}\left(\tau_{-h}\right)\right) \equiv$ $\beta\left(u_{2}\left(\tau_{-h}\right)\right)$ on $\llbracket t, S\left(\tau_{-h}\right) \rrbracket$. Therefore,

$$
\beta^{h}\left(u_{1}\right)=\beta\left(u_{1}\left(\tau_{-h}\right)\right)\left(\tau_{h}\right) \equiv \beta\left(u_{2}\left(\tau_{-h}\right)\right)\left(\tau_{h}\right)=\beta^{h}\left(u_{2}\right) \text { on } \llbracket t, S \rrbracket .
$$

$3^{\text {rd }}$ step: For all $h \in H_{t}$ and $\beta \in \mathcal{B}_{t, T}$ we have:

$$
\left\{\operatorname{esssup}_{u \in \mathcal{U}_{t, T}} J(t, x ; u, \beta(u))\right\}\left(\tau_{h}\right)=\operatorname{esssup}_{u \in \mathcal{U}_{t, T}}\left\{J(t, x ; u, \beta(u))\left(\tau_{h}\right)\right\} \text {, P-a.s. }
$$

Indeed, with the notation $I(t, x, \beta):=\operatorname{esssup}_{u \in \mathcal{U}_{t, T}} J(t, x ; u, \beta(u)), \beta \in \mathcal{B}_{t, T}$, we have $I(t, x, \beta) \geq J(t, x ; u, \beta(u))$, and thus $I(t, x, \beta)\left(\tau_{h}\right) \geq J(t, x ; u, \beta(u))\left(\tau_{h}\right)$, P-a.s., for all $u \in \mathcal{U}_{t, T}$. On the other hand, for any random variable $\zeta$ satisfying $\zeta \geq J(t, x ; u, \beta(u))\left(\tau_{h}\right)$ and hence also $\zeta\left(\tau_{-h}\right) \geq J(t, x ; u, \beta(u))$, P-a.s., for all $u \in$ 
$\mathcal{U}_{t, T}$, we have $\zeta\left(\tau_{-h}\right) \geq I(t, x, \beta)$, P-a.s., i.e., $\zeta \geq I(t, x, \beta)\left(\tau_{h}\right)$, P-a.s. Consequently,

$$
I(t, x, \beta)\left(\tau_{h}\right)=\operatorname{esssup}_{u \in \mathcal{U}_{t, T}}\left\{J(t, x ; u, \beta(u))\left(\tau_{h}\right)\right\}, \text { P-a.s. }
$$

4th step: $W(t, x)$ is invariant with respect to the Girsanov transformation $\tau_{h}$, i.e.,

$$
W(t, x)\left(\tau_{h}\right)=W(t, x), \text { P-a.s., for any } h \in H .
$$

Let us first assume that $h \in H_{t}$. Then, similarly to the third step we can show that for all $h \in H_{t}$,

$$
\left\{\operatorname{essinf}_{\beta \in \mathcal{B}_{t, T}} I(t, x ; \beta)\right\}\left(\tau_{h}\right)=\operatorname{essinf}_{\beta \in \mathcal{B}_{t, T}}\left\{I(t, x ; \beta)\left(\tau_{h}\right)\right\} \text {, P-a.s. }
$$

Then, using the results of the former three steps we have, for any $h \in H_{t}$,

$$
\begin{aligned}
W(t, x)\left(\tau_{h}\right) & =\operatorname{essinf}_{\beta \in \mathcal{B}_{t, T}} \operatorname{esssup}_{u \in \mathcal{U}_{t, T}}\left\{J(t, x ; u, \beta(u))\left(\tau_{h}\right)\right\} \\
& =\operatorname{essinf}_{\beta \in \mathcal{B}_{t, T}} \operatorname{essup}_{u \in \mathcal{U}_{t, T}} J\left(t, x ; u\left(\tau_{h}\right), \beta^{h}\left(u\left(\tau_{h}\right)\right)\right) \\
& =\operatorname{essinf}_{\beta \in \mathcal{B}_{t, T}} \operatorname{essup}_{u \in \mathcal{U}_{t, T}} J\left(t, x ; u, \beta^{h}(u)\right) \\
& =\operatorname{essinf}_{\beta \in \mathcal{B}_{t, T}} \operatorname{esssup}_{u \in \mathcal{U}_{t, T}} J(t, x ; u, \beta(u)) \\
& =W(t, x), \text { P-a.s., }
\end{aligned}
$$

where we have used the relations $\left\{u\left(\tau_{h}\right) \mid u(\cdot) \in \mathcal{U}_{t, T}\right\}=\mathcal{U}_{t, T},\left\{\beta^{h} \mid \beta \in \mathcal{B}_{t, T}\right\}=$ $\mathcal{B}_{t, T}$ in order to obtain the both latter equalities. Therefore, for any $h \in H_{t}, W(t, x)$ $\left(\tau_{h}\right)=W(t, x), \mathrm{P}$-a.s., and since $W(t, x)$ is $\mathcal{F}_{t}$-measurable, we have this relation even for all $h \in H$. Indeed, recall that our underlying fundamental space is $\Omega=C_{0}\left([0, T] ; \mathbb{R}^{d}\right)$ and that, due to the definition of the filtration, the $\mathcal{F}_{t}$-measurable random variable $W(t, x)(\omega), \omega \in \Omega$, depends only on the restriction of $\omega$ to the time interval $[0, t]$.

The result of the 4th step combined with the following auxiliary Lemma 3.1 completes our proof.

Lemma 3.1. Let $\zeta$ be a random variable defined over our classical Wiener space $\left(\Omega, \mathcal{F}_{T}, P\right)$, such that $\zeta\left(\tau_{h}\right)=\zeta$, P-a.s., for any $h \in H$. Then $\zeta=E \zeta$, P-a.s.

For the proof the reader is referred, for instance, to Lemma 3.4 in Buckdahn and $\mathrm{Li}[3]$.

The first property of the lower value function $W(t, x)$ which we present below is an immediate consequence of (3.6) and (3.9).

Lemma 3.2. There exists a constant $C>0$ such that, for all $0 \leq t \leq T, x, x^{\prime} \in \mathbb{R}^{n}$,

(i) $\left|W(t, x)-W\left(t, x^{\prime}\right)\right| \leq C\left|x-x^{\prime}\right|$;

(ii) $|W(t, x)| \leq C(1+|x|)$.

We now discuss (the generalized) DPP for our stochastic differential game with reflection (3.1), (3.5) and (3.9). For this end we have to define the family of (backward) semigroups associated with RBSDE (3.5). This notion of stochastic backward semigroups was first introduced by Peng [25] and applied to study the DPP for stochastic control problems. Our approach adapts Peng's ideas to the framework of stochastic differential games with reflection. 
Given the initial data $(t, x)$, a positive number $\delta \leq T-t$, admissible control processes $u(\cdot) \in \mathcal{U}_{t, t+\delta}, v(\cdot) \in \mathcal{V}_{t, t+\delta}$ and a real-valued random variable $\eta \in L^{2}\left(\Omega, \mathcal{F}_{t+\delta}, P ; \mathbb{R}\right)$ such that $h\left(t+\delta, X_{t+\delta}^{t, x ; u, v}\right) \leq \eta \leq h^{\prime}\left(t+\delta, X_{t+\delta}^{t, x ; u, v}\right)$, a.s., we put

$$
G_{s, t+\delta}^{t, x ; u, v}[\eta]:=\tilde{Y}_{s}^{t, x ; u, v}, \quad s \in[t, t+\delta],
$$

where $\left(\tilde{Y}_{s}^{t, x ; u, v}, \tilde{Z}_{s}^{t, x ; u, v}, \tilde{K}_{s}^{+, t, x ; u, v}, \tilde{K}_{s}^{-, t, x ; u, v}\right)_{t<s<t+\delta}$ is the solution of the following RBSDE with time horizon $t+\delta$ :

(i) $\tilde{Y}^{t, x ; u, v} \in \mathcal{S}^{2}(t, t+\delta ; \mathbb{R}), \tilde{Z}^{t, x ; u, v} \in \mathcal{H}\left(t, t+\delta ; \mathbb{R}^{d}\right)$, and $\tilde{K}^{+, t, x ; u, v}$,

$$
\tilde{K}^{-, t, x ; u, v} \in \mathcal{A}_{c}(t, t+\delta ; \mathbb{R}) ;
$$

(ii) $\tilde{Y}_{s}^{t, x ; u, v}=\eta+\int_{s}^{t+\delta} f\left(r, X_{r}^{t, x ; u, v}, \tilde{Y}_{r}^{t, x ; u, v}, \tilde{Z}_{r}^{t, x ; u, v}, u_{r}, v_{r}\right) d r$

$$
\begin{aligned}
& +\left(\tilde{K}_{t+\delta}^{+, t, x ; u, v}-\tilde{K}_{s}^{+, t, x ; u, v}\right)-\left(\tilde{K}_{t+\delta}^{-, t, x ; u, v}-\tilde{K}_{s}^{-, t, x ; u, v}\right) \\
& -\int_{s}^{t+\delta} \tilde{Z}_{r}^{t, x ; u, v} d B_{r}, \quad s \in[t, t+\delta] ;
\end{aligned}
$$

(iii) $h\left(s, X_{s}^{t, x ; u, v}\right) \leq \tilde{Y}_{s}^{t, x ; u, v} \leq h^{\prime}\left(s, X_{s}^{t, x ; u, v}\right)$, a.s., for any $s \in[t, t+\delta]$;

$$
\begin{gathered}
\text { (iv) } \int_{t}^{t+\delta}\left(\tilde{Y}_{r}^{t, x ; u, v}-h\left(r, X_{r}^{t, x ; u, v}\right)\right) d \tilde{K}_{r}^{+, t, x ; u, v}=\int_{t}^{t+\delta}\left(h^{\prime}\left(r, X_{r}^{t, x ; u, v}\right)\right. \\
\left.-\tilde{Y}_{r}^{t, x ; u, v}\right) d \tilde{K}_{r}^{-, t, x ; u, v}=0,
\end{gathered}
$$

where $X^{t, x ; u, v}$ is introduced by Eq. (3.1).

Then, in particular, for the solution $\left(Y^{t, x ; u, v}, Z^{t, x ; u, v}, K^{+, t, x ; u, v}, K^{-, t, x ; u, v}\right)$ of the RBSDE with two barriers (3.5) we have

$$
G_{t, T}^{t, x ; u, v}\left[\Phi\left(X_{T}^{t, x ; u, v}\right)\right]=G_{t, t+\delta}^{t, x ; u, v}\left[Y_{t+\delta}^{t, x ; u, v}\right] .
$$

Moreover,

$$
\begin{aligned}
J(t, x ; u, v) & =Y_{t}^{t, x ; u, v}=G_{t, T}^{t, x ; u, v}\left[\Phi\left(X_{T}^{t, x ; u, v}\right)\right]=G_{t, t+\delta}^{t, x ; u, v}\left[Y_{t+\delta}^{t, x ; u, v}\right] \\
& =G_{t, t+\delta}^{t, x ; u, v}\left[J\left(t+\delta, X_{t+\delta}^{t, x ; u, v} ; u, v\right)\right],
\end{aligned}
$$

where the latter equality follows from (3.8) and the relation

$$
Y_{t+\delta}^{t, x, u, v}=Y_{t+\delta}^{t+\delta, X_{t+\delta}^{t, x, u, v}, u, v}
$$

a consequence of the uniqueness of the solution of BSDE (3.5) and that of the associated forward Eq. (3.1). In particular, we have

$$
W(t, x)=\operatorname{essinf}_{\beta \in \mathcal{B}_{t, T}} \operatorname{esssup}_{u \in \mathcal{U}_{t, T}} G_{t, T}^{t, x ; u, \beta(u)}\left[\Phi\left(X_{T}^{t, x ; u, \beta(u)}\right)\right] .
$$

Remark 3.2. For the better comprehension for the reader let us point out that if $f$ is independent of $(y, z)$ then, for all $s \in[t, t+\delta]$,

$$
\begin{aligned}
G_{s, t+\delta}^{t, x ; u, v}[\eta]= & E\left[\eta+\int_{s}^{t+\delta} f\left(r, X_{r}^{t, x ; u, v}, u_{r}, v_{r}\right) d r+\left(\tilde{K}_{t+\delta}^{+, t, x ; u, v}-\tilde{K}_{s}^{+, t, x ; u, v}\right)\right. \\
& \left.-\left(\tilde{K}_{t+\delta}^{-, t, x ; u, v}-\tilde{K}_{s}^{-, t, x ; u, v}\right) \mid \mathcal{F}_{s}\right] .
\end{aligned}
$$


Theorem 3.1. Under the assumptions (H3.1) and (H3.2), the lower value function $W(t, x)$ obeys the following DPP : For any $0 \leq t<t+\delta \leq T, x \in \mathbb{R}^{n}$,

$$
W(t, x)=\operatorname{essinf}_{\beta \in \mathcal{B}_{t, t+\delta}} \operatorname{esssup}_{u \in \mathcal{U}_{t, t+\delta}} G_{t, t+\delta}^{t, x ; u, \beta(u)}\left[W\left(t+\delta, X_{t+\delta}^{t, x ; u, \beta(u)}\right)\right] .
$$

The proof of Theorem 3.1 is given in the appendix.

Remark 3.3. We emphasize that, unlike Buckdahn and Li [3,4], here we won't use DPP to prove that $W$ and $U$ are the viscosity solutions of the associated Isaacs with two barriers, respectively.

\section{Viscosity solution of Isaacs equation with obstacles: existence theorem}

In this section we consider the following Isaacs equations with obstacles

$$
\left\{\begin{array}{l}
\min \left\{W(t, x)-h(t, x), \max \left[-\frac{\partial}{\partial t} W(t, x)-H^{-}\left(t, x, W, D W, D^{2} W\right)\right.\right. \\
\left.\left.\quad W(t, x)-h^{\prime}(t, x)\right]\right\}=0 \\
W(T, x)=\Phi(x)
\end{array}\right.
$$

and

$$
\left\{\begin{array}{l}
\min \left\{U(t, x)-h(t, x), \max \left[-\frac{\partial}{\partial t} U(t, x)-H^{+}\left(t, x, U, D U, D^{2} U\right),\right.\right. \\
\left.\left.\quad W(t, x)-h^{\prime}(t, x)\right]\right\}=0, \\
U(T, x)=\Phi(x)
\end{array}\right.
$$

associated with the Hamiltonians

$$
H^{-}(t, x, y, q, X)=\sup _{u \in U} \inf _{v \in V}\left\{\frac{1}{2} \operatorname{tr}\left(\sigma \sigma^{T} X\right)+q . b+f(t, x, y, q . \sigma, u, v)\right\}
$$

and

$$
H^{+}(t, x, y, q, X)=\inf _{v \in V} \sup _{u \in U}\left\{\frac{1}{2} \operatorname{tr}\left(\sigma \sigma^{T} X\right)+q . b+f(t, x, y, q . \sigma, u, v)\right\},
$$

respectively, where $\sigma$ stands for $\sigma(t, x, u, v), b$ for $b(t, x, u, v)$, and $t \in[0, T], x \in$ $\mathbb{R}^{n}, y \in \mathbb{R}, q \in \mathbb{R}^{n}$ and $X \in \mathbb{S}^{n}$ (Recall that $\mathbb{S}^{n}$ denotes the set of symmetric $n \times n$-matrices. Here the functions $b, \sigma, f, h, h^{\prime}$ and $\Phi$ are supposed to satisfy (H3.1) and (H3.2), respectively.

In this section we want to prove that the lower value function $W(t, x)$ introduced by (3.9) is the viscosity solution of Eq. (4.1), while the upper value function $U(t, x)$ defined by (3.10) is the viscosity solution of Eq. (4.2). The uniqueness of the viscosity solution will be shown in the next section for the class of continuous functions satisfying some growth assumption which is weaker than the polynomial growth condition. We first recall the definition of a viscosity solution of Eq. (4.1), that for Eq. (4.2) is similar. We borrow the definition from Crandall, Ishii and Lions [5]. 
Defnition 4.1. (i) A real-valued upper semicontinuous function $W:[0, T] \times$ $\mathbb{R}^{n} \rightarrow \mathbb{R}$ is called a viscosity subsolution of Eq. (4.1) if $W(T, x) \leq \Phi(x)$, for all $x \in \mathbb{R}^{n}$, and if for all functions $\varphi \in C_{l, b}^{3}\left([0, T] \times \mathbb{R}^{n}\right)$ and $(t, x) \in[0, T) \times \mathbb{R}^{n}$ such that $W-\varphi$ attains a local maximum at $(t, x)$, we have

$$
\begin{aligned}
\min & \{W(t, x)-h(t, x), \\
& \left.\max \left[-\frac{\partial \varphi}{\partial t}(t, x)-H^{-}\left(t, x, W, D \varphi, D^{2} \varphi\right), W(t, x)-h^{\prime}(t, x)\right]\right\} \leq 0 ;
\end{aligned}
$$

(ii) A real-valued lower semicontinuous function $W:[0, T] \times \mathbb{R}^{n} \rightarrow \mathbb{R}$ is called a viscosity supersolution of Eq. (4.1) if $W(T, x) \geq \Phi(x)$, for all $x \in \mathbb{R}^{n}$, and if for all functions $\varphi \in C_{l, b}^{3}\left([0, T] \times \mathbb{R}^{n}\right)$ and $(t, x) \in[0, T) \times \mathbb{R}^{n}$ such that $W-\varphi$ attains a local minimum at $(t, x)$, it holds

$$
\begin{aligned}
\min & \{W(t, x)-h(t, x), \\
& \left.\max \left[-\frac{\partial \varphi}{\partial t}(t, x)-H^{-}\left(t, x, W, D \varphi, D^{2} \varphi\right), W(t, x)-h^{\prime}(t, x)\right]\right\} \geq 0 ;
\end{aligned}
$$

(iii) A real-valued continuous function $W \in C\left([0, T] \times \mathbb{R}^{n}\right)$ is called a viscosity solution of Eq. (4.1) if it is both a viscosity sub- and a supersolution of Eq. (4.1).

Remark 4.1. $\quad C_{l, b}^{3}\left([0, T] \times \mathbb{R}^{n}\right)$ denotes the set of the real-valued functions that are continuously differentiable up to the third order and whose derivatives of the orders 1, 2 and 3 are bounded.

We now state the main result of this section.

Theorem 4.1. Under the assumptions (H3.1) and (H3.2) the lower value function $W$ defined by (3.9) is a viscosity solution of the Isaacs equation with two barriers (4.1), while $U$ defined by (3.10) solves the Isaacs equation with two barriers (4.2) in the viscosity solution sense.

We will develop the proof of this theorem only for $W$, that of $U$ is analogous. The proof is mainly based on an approximation of our RBSDE (3.5) by a sequence of penalized BSDEs with one barrier. This penalization method for RBSDEs was first studied in [9], Sect. 6 (pp. 719-723).

For each $(t, x) \in[0, T] \times \mathbb{R}^{n}$, and $m \in \mathbf{N}$, let $\left({ }^{m} Y_{s}^{t, x ; u, v}\right)_{t \leq s \leq T}$ (respectively, $\left.\left({ }^{m} \bar{Y}_{s}^{t, x ; u, v}\right)_{t \leq s \leq T}\right)$ be the first component of the unique solution of the BSDE with one reflecting lower (resp., upper) barrier associated with $\left(f\left(r, X_{r}^{t, x ; u, v}, y, z\right.\right.$, $\left.\left.u_{r}, v_{r}\right)-m\left(h^{\prime}\left(r, X_{r}^{t, x ; u, v}\right)-y\right)^{-}, \Phi\left(X_{T}^{t, x ; u, v}\right), h\left(r, X_{r}^{t, x ; u, v}\right)\right) \quad$ (respectively, $\left.\left(f\left(r, X_{r}^{t, x ; u, v}, y, z, u_{r}, v_{r}\right)+m\left(y-h\left(r, X_{r}^{t, x ; u, v}\right)\right)^{-}, \Phi\left(X_{T}^{t, x ; u, v}\right), h^{\prime}\left(r, X_{r}^{t, x ; u, v}\right)\right)\right)$ (recall that the solutions ${ }^{m} Y^{t, x ; u, v}$ and ${ }^{m} \bar{Y}^{t, x ; u, v}$ exist due to Lemma 2.3). We define

$$
J_{m}(t, x ; u, v):={ }^{m} Y_{t}^{t, x ; u, v}, \quad u \in \mathcal{U}_{t, T}, \quad v \in \mathcal{V}_{t, T}, 0 \leq t \leq T, x \in \mathbb{R}^{n},
$$


and associate the lower value function

$$
W_{m}(t, x):=\operatorname{essinf}_{\beta \in \mathcal{B}_{t, T}} \operatorname{esssup}_{u \in \mathcal{U}_{t, T}} J_{m}(t, x ; u, \beta(u)), \quad 0 \leq t \leq T, x \in \mathbb{R}^{n} .
$$

(respectively,

$$
\bar{J}_{m}(t, x ; u, v):={ }^{m} \bar{Y}_{t}^{t, x ; u, v}, \quad u \in \mathcal{U}_{t, T}, \quad v \in \mathcal{V}_{t, T}, 0 \leq t \leq T, x \in \mathbb{R}^{n},
$$

for which we consider the lower value function

$$
\left.\bar{W}_{m}(t, x):=\operatorname{essinf}_{\beta \in \mathcal{B}_{t, T}} \operatorname{esssup}_{u \in \mathcal{U}_{t, T}} \bar{J}_{m}(t, x ; u, \beta(u)), \quad 0 \leq t \leq T, x \in \mathbb{R}^{n} .\right)
$$

It is known from Buckdahn and $\mathrm{Li}[4]$ that $W_{m}(t, x)$ defined in (4.4) is in $C\left([0, T] \times \mathbb{R}^{n}\right)$, has linear growth in $x$, and is a continuous viscosity solution of the following Isaacs equation with one barrier:

$$
\left\{\begin{array}{l}
\min \left\{W_{m}(t, x)-h(t, x),-\frac{\partial}{\partial t} W_{m}(t, x)-\sup _{u \in U} \inf _{v \in V}\left\{\frac { 1 } { 2 } \operatorname { t r } \left(\sigma \sigma^{T}(t, x, u, v)\right.\right.\right. \\
\left.\quad \times D^{2} W_{m}(t, x)\right)+D W_{m}(t, x) . b(t, x, u, v)+f_{m}\left(t, x, W_{m}(t, x), D W_{m}(t, x) .\right. \\
\sigma(t, x, u, v), u, v)\}\}=0, \\
W_{m}(T, x)=\Phi(x), \quad x \in \mathbb{R}^{n},
\end{array}\right.
$$

where

$$
\begin{aligned}
f_{m}(t, x, y, z, u, v)= & f(t, x, y, z, u, v)-m\left(h^{\prime}(t, x)-y\right)^{-}, \\
& (t, x, y, z, u, v) \in[0, T] \times \mathbb{R}^{n} \times \mathbb{R} \times \mathbb{R}^{d} \times U \times V .
\end{aligned}
$$

Also the function $\bar{W}_{m}(t, x)$ defined in $(4.6)$ is in $C\left([0, T] \times \mathbb{R}^{n}\right)$, has linear growth in $x$, and is a continuous viscosity solution of the following Isaacs equation with one barrier:

$$
\left\{\begin{array}{l}
\max \left\{\bar{W}_{m}(t, x)-h^{\prime}(t, x),-\frac{\partial}{\partial t} \bar{W}_{m}(t, x)-\sup _{u \in U} \inf _{v \in V}\left\{\frac { 1 } { 2 } \operatorname { t r } \left(\sigma \sigma^{T}(t, x, u, v)\right.\right.\right. \\
\left.\quad \times D^{2} \bar{W}_{m}(t, x)\right)+D \bar{W}_{m}(t, x) \cdot b(t, x, u, v)+\bar{f}_{m}\left(t, x, \bar{W}_{m}(t, x), D \bar{W}_{m}(t, x) .\right. \\
\sigma(t, x, u, v), u, v)\}\}=0, \\
\bar{W}_{m}(T, x)=\Phi(x), \quad x \in \mathbb{R}^{n},
\end{array}\right.
$$

where

$$
\begin{aligned}
\bar{f}_{m}(t, x, y, z, u, v)= & f(t, x, y, z, u, v)+m(y-h(t, x))^{-}, \\
& (t, x, y, z, u, v) \in[0, T] \times \mathbb{R}^{n} \times \mathbb{R} \times \mathbb{R}^{d} \times U \times V .
\end{aligned}
$$

We have the uniqueness of the viscosity solutions $W_{m}, \bar{W}_{m}$ in the space $\tilde{\Theta}$ which is defined by $\tilde{\Theta}=\left\{\varphi \in C\left([0, T] \times \mathbb{R}^{n}\right): \exists \widetilde{A}>0\right.$ such that

$$
\left.\lim _{|x| \rightarrow \infty} \varphi(t, x) \exp \left\{-\widetilde{A}\left[\log \left(\left(|x|^{2}+1\right)^{\frac{1}{2}}\right)\right]^{2}\right\}=0 \text {, uniformly in } t \in[0, T]\right\} .
$$

Lemma 4.1. For all $(t, x) \in[0, T] \times \mathbb{R}^{n}$ and all $m \geq 1$,

$$
\begin{aligned}
& W_{1}(t, x) \geq \cdots \geq W_{m}(t, x) \geq W_{m+1}(t, x) \geq \cdots \geq W(t, x) . \\
& \bar{W}_{1}(t, x) \leq \cdots \leq \bar{W}_{m}(t, x) \leq \bar{W}_{m+1}(t, x) \leq \cdots \leq W(t, x) .
\end{aligned}
$$


Proof. Let $m \geq 1$, since $f_{m}(t, x, y, z, u, v) \geq f_{m+1}(t, x, y, z, u, v)$, for all $(t, x, y$, $z, u, v)$ we obtain from the comparison theorem for BSDEs with one barrier (Lemma 2.4) that

$$
\begin{aligned}
& J_{m}(t, x, u, v)={ }^{m} Y_{t}^{t, x ; u, v} \geq{ }^{m+1} Y_{t}^{t, x ; u, v}=J_{m+1}(t, x, u, v), \\
& \text { P-a.s., for any } u \in \mathcal{U}_{t, T} \text { and } v \in \mathcal{V}_{t, T} .
\end{aligned}
$$

Consequently, $W_{m}(t, x) \geq W_{m+1}(t, x)$, for all $(t, x) \in[0, T] \times \mathbb{R}^{n}, m \geq 1$.

From the comparison principle of Sect. 3 [pp.247-256] in [18] we get that, for each $0 \leq t \leq T, x \in \mathbb{R}^{n}, u \in \mathcal{U}_{t, T}$ and $v \in \mathcal{V}_{t, T}$,

$$
J_{m}(t, x ; u, v) \geq J(t, x ; u, v), \text { P-a.s. }
$$

It follows that $W_{m}(t, x) \geq W(t, x)$, for all $(t, x) \in[0, T] \times \mathbb{R}^{n}, m \geq 1$. The proof for $\bar{W}_{m}(t, x)$ can be carried out in a similar way.

Remark 4.2. The above lemma allows to introduce the upper semicontinuous function $\widetilde{W}$ as limit over the non-increasing sequence of continuous functions $W_{m}, m \geq 1$, and we have

$$
W_{1}(t, x) \geq \widetilde{W}(t, x)\left(=\lim _{m \uparrow \infty} \downarrow W_{m}(t, x)\right) \geq W(t, x), \quad(t, x) \in[0, T] \times \mathbb{R}^{n} .
$$

Remark 4.3. The above lemma also allows to introduce the lower semicontinuous function $\widetilde{\widetilde{W}}$ as limit over the non-decreasing sequence of continuous functions $\bar{W}_{m}, m \geq 1$. From

$$
\bar{W}_{1}(t, x) \leq \widetilde{\bar{W}}(t, x)\left(=\lim _{m \uparrow \infty} \uparrow \bar{W}_{m}(t, x)\right) \leq W(t, x), \quad(t, x) \in[0, T] \times \mathbb{R}^{n},
$$

and from Remark 4.2 and the linear growth of $\bar{W}_{1}$ and $W_{1}$ we conclude that also $\widetilde{W}$ and $\widetilde{W}$ have at most linear growth.

Our objective is to prove that $\widetilde{W}, \widetilde{\bar{W}}$ and $W$ coincide and Eq. (4.1) holds in viscosity sense. For this end we first prove the following proposition:

Proposition 4.1. Under the assumptions (H3.1) and (H3.2) the function $\widetilde{W}(t, x)$ is a viscosity subsolution of Isaacs Eq. (4.1).

Proof. Let $(t, x) \in[0, T) \times \mathbb{R}^{n}$ and let $\varphi \in C_{l, b}^{3}\left([0, T] \times \mathbb{R}^{n}\right)$ be such that $\widetilde{W}-\varphi<$ $\widetilde{W}(t, x)-\varphi(t, x)$ everywhere on $\left([0, T] \times \mathbb{R}^{n}\right)-\{(t, x)\}$. Then, since $\widetilde{W}$ is upper semicontinuous and $W_{m}(t, x) \downarrow \widetilde{W}(t, x), 0 \leq t \leq T, x \in \mathbb{R}^{n}$, there exists some sequence $\left(t_{m}, x_{m}\right), m \geq 1$, such that, at least along a subsequence,

(i) $\left(t_{m}, x_{m}\right) \rightarrow(t, x)$, as $m \rightarrow+\infty$;

(ii) $W_{m}-\varphi \leq W_{m}\left(t_{m}, x_{m}\right)-\varphi\left(t_{m}, x_{m}\right)$ in a neighborhood of $\left(t_{m}, x_{m}\right)$, for all $m \geq 1$

(iii) $W_{m}\left(t_{m}, x_{m}\right) \rightarrow \widetilde{W}(t, x)$, as $m \rightarrow+\infty$. 
From the definition of $\widetilde{W}(t, x)$ we know $\widetilde{W}(t, x) \geq h(t, x)$. Therefore we only need to distinguish two cases. In the case, for which $\widetilde{W}(t, x)=h(t, x)$, Eq. $\left(4.1^{\prime}\right)$ is trivially satisfied, and the proof is complete. Let us discuss the second case: $\widetilde{W}(t, x)>h(t, x)$. For this case we get as an immediate consequence of i) and iii) the following result:

(iv) There exists $N$ such that $W_{m}\left(t_{m}, x_{m}\right)>h\left(t_{m}, x_{m}\right)$, for all $m>N$.

Thus, because $W_{m}$ is a viscosity solution and hence a subsolution of Eq. (4.7), we have, for all $m \geq 1$,

$$
\begin{aligned}
& \frac{\partial}{\partial t} \varphi\left(t_{m}, x_{m}\right)+\sup _{u \in U} \inf _{v \in V}\left\{\frac{1}{2} \operatorname{tr}\left(\sigma \sigma^{*}\left(t_{m}, x_{m}, u, v\right) D^{2} \varphi\left(t_{m}, x_{m}\right)\right)\right. \\
& \quad+b\left(t_{m}, x_{m}, u, v\right) D \varphi\left(t_{m}, x_{m}\right)+f\left(t_{m}, x_{m}, W_{m}\left(t_{m}, x_{m}\right)\right. \\
& \left.\left.\quad D \varphi\left(t_{m}, x_{m}\right) \sigma\left(t_{m}, x_{m}, u, v\right), u, v\right)\right\} \\
& \quad-m\left(h^{\prime}\left(t_{m}, x_{m}\right)-W_{m}\left(t_{m}, x_{m}\right)\right)^{-} \\
& \geq 0
\end{aligned}
$$

Therefore,

$$
\begin{aligned}
& \frac{\partial}{\partial t} \varphi\left(t_{m}, x_{m}\right)+\sup _{u \in U} \inf _{v \in V}\left\{\frac{1}{2} \operatorname{tr}\left(\sigma \sigma^{*}\left(t_{m}, x_{m}, u, v\right) D^{2} \varphi\left(t_{m}, x_{m}\right)\right)\right. \\
& \quad+b\left(t_{m}, x_{m}, u, v\right) D \varphi\left(t_{m}, x_{m}\right)+f\left(t_{m}, x_{m}, W_{m}\left(t_{m}, x_{m}\right)\right. \\
& \left.\left.\quad D \varphi\left(t_{m}, x_{m}\right) \sigma\left(t_{m}, x_{m}, u, v\right), u, v\right)\right\} \\
& \geq 0 .
\end{aligned}
$$

We recall that $\left(t_{m}, x_{m}\right) \rightarrow(t, x)$ and $W_{m}\left(t_{m}, x_{m}\right) \rightarrow \widetilde{W}(t, x)$, as $m \rightarrow+\infty$. On the other hand, from the continuity of the functions $b, \sigma$ and $f$ we have, in particular, their uniform continuity on compacts (recall that $U, V$ are compacts). Consequently,

$$
\begin{aligned}
& \frac{\partial}{\partial t} \varphi\left(t_{m}, x_{m}\right)+\frac{1}{2} \operatorname{tr}\left(\sigma \sigma^{*}\left(t_{m}, x_{m}, u, v\right) D^{2} \varphi\left(t_{m}, x_{m}\right)\right) \\
& +b\left(t_{m}, x_{m}, u, v\right) D \varphi\left(t_{m}, x_{m}\right)+f\left(t_{m}, x_{m}, W_{m}\left(t_{m}, x_{m}\right),\right. \\
& \left.\quad D \varphi\left(t_{m}, x_{m}\right) \sigma\left(t_{m}, x_{m}, u, v\right), u, v\right)
\end{aligned}
$$

converges, uniformly in $(u, v)$, towards

$$
\begin{gathered}
\frac{\partial}{\partial t} \varphi(t, x)+\frac{1}{2} \operatorname{tr}\left(\sigma \sigma^{*}(t, x, u, v) D^{2} \varphi(t, x)\right) \\
+b(t, x, u, v) D \varphi(t, x)+f(t, x, \widetilde{W}(t, x) \\
D \varphi(t, x) \sigma(t, x, u, v), u, v) .
\end{gathered}
$$


Therefore,

$$
\begin{aligned}
& \frac{\partial}{\partial t} \varphi(t, x)+\sup _{u \in U} \inf _{v \in V}\left\{\frac{1}{2} \operatorname{tr}\left(\sigma \sigma^{*}(t, x, u, v) D^{2} \varphi(t, x)\right)\right. \\
& \quad+b(t, x, u, v) D \varphi(t, x)+f(t, x, \widetilde{W}(t, x), D \varphi(t, x) \sigma(t, x, u, v), u, v)\} \\
& \quad \geq 0 .
\end{aligned}
$$

The above calculation shows that if $\widetilde{W}(t, x) \leq h^{\prime}(t, x)$ then we can conclude $\widetilde{W}$ is a viscosity subsolution of (4.1). For proving that $\widetilde{W}(t, x) \leq h^{\prime}(t, x)$ we return to the above inequality (4.10), from where

$$
\begin{aligned}
& m\left(h^{\prime}\left(t_{m}, x_{m}\right)-W_{m}\left(t_{m}, x_{m}\right)\right)^{-} \\
& \leq \frac{\partial}{\partial t} \varphi\left(t_{m}, x_{m}\right)+\sup _{u \in U} \inf _{v \in V}\left\{\frac{1}{2} \operatorname{tr}\left(\sigma \sigma^{*}\left(t_{m}, x_{m}, u, v\right) D^{2} \varphi\left(t_{m}, x_{m}\right)\right)\right. \\
& \quad+b\left(t_{m}, x_{m}, u, v\right) D \varphi\left(t_{m}, x_{m}\right)+f\left(t_{m}, x_{m}, W_{m}\left(t_{m}, x_{m}\right),\right. \\
&\left.\left.\quad D \varphi\left(t_{m}, x_{m}\right) \sigma\left(t_{m}, x_{m}, u, v\right), u, v\right)\right\} .
\end{aligned}
$$

When $m$ tends to $+\infty$ the limit of the right-hand side of the above inequality, given by the left hand side of (4.11), is a real number. Therefore, the left-hand side of the above inequality cannot tend to $+\infty$. But this is only possible if $\left(h^{\prime}\left(t_{m}, x_{m}\right)-W_{m}\left(t_{m}, x_{m}\right)\right)^{-} \rightarrow 0$, i.e., if $\widetilde{W}(t, x) \leq h^{\prime}(t, x)$. The proof is complete.

Proposition 4.2. Under the assumptions (H3.1) and (H3.2) the function $\widetilde{\bar{W}}(t, x)$ is a viscosity supersolution of Isaacs equations (4.1).

The proof is similar to that of Proposition 4.1, so we omit it.

Proof of Theorem 4.1. From Theorem 5.1 which is proved in Sect. 5, Propositions 4.1 and 4.2 we get $\widetilde{W}(t, x) \leq \widetilde{W}(t, x)$. Furthermore, from Remark 4.2 and Remark 4.3 we get $\widetilde{W}(t, x)=\widetilde{W}(t, x)=W(t, x)$. The proof is complete.

As a byproduct to the proof of Theorem 5.1 we have that both the viscosity solution $W_{m}$ of the Isaacs equation with one obstacle (4.7) and the viscosity solution $\bar{W}_{m}$ of the Isaacs equation with one obstacle (4.8) converge pointwise to the viscosity solution of the Isaacs equation with two obstacles (4.1) :

Theorem 4.2. $W_{m}(t, x) \downarrow W(t, x)$ and $\bar{W}_{m}(t, x) \uparrow W(t, x)$, as $m \rightarrow+\infty$, for any $(t, x) \in[0, T] \times \mathbb{R}^{n}$.

On the other hand, we can also describe $W$ as limit of solutions of a sequence of Bellman-Isaacs equations without obstacle. For this end we let, for each $(t, x) \in[0, T] \times \mathbb{R}^{n}$, and $m, n \in \mathbf{N},\left({ }^{m, n} Y_{s}^{t, x ; u, v}\right)_{t \leq s \leq T}$ be the first component of the unique solution of the BSDE associated with $\left(\bar{f}\left(r, X_{r}^{t, x ; u, v}, y, z, u_{r}, v_{r}\right)-\right.$ $\left.m\left(h^{\prime}\left(r, X_{r}^{t, x ; u, v}\right)-y\right)^{-}+n\left(y-h\left(r, X_{r}^{t, x ; u, v}\right)\right)^{-}, \Phi\left(X_{T}^{t, x ; u, v}\right)\right)$ (recall that due to Lemma $2.1^{m, n} Y^{t, x ; u, v}$ exists). We define

$$
J_{m, n}(t, x ; u, v):=^{m, n} Y_{t}^{t, x ; u, v}, \quad u \in \mathcal{U}_{t, T}, \quad v \in \mathcal{V}_{t, T}, 0 \leq t \leq T, x \in \mathbb{R}^{n},
$$


and consider the lower value function

$W_{m, n}(t, x):=\operatorname{essinf}_{\beta \in \mathcal{B}_{t, T}} \operatorname{esssup}_{u \in \mathcal{U}_{t, T}} J_{m, n}(t, x ; u, \beta(u)), \quad 0 \leq t \leq T, x \in \mathbb{R}^{n}$.

It is known from Buckdahn and $\mathrm{Li}[3]$ that $W_{m, n}(t, x)$ defined in (4.13) is in $C\left([0, T] \times \mathbb{R}^{n}\right)$, has linear growth in $x$, and is the unique continuous viscosity solution of the following Isaacs equations:

$$
\left\{\begin{array}{l}
-\frac{\partial}{\partial t} W_{m, n}(t, x)-\sup _{u \in U} \inf _{v \in V}\left\{\frac{1}{2} \operatorname{tr}\left(\sigma \sigma^{T}(t, x, u, v) D^{2} W_{m, n}(t, x)\right)\right. \\
\quad+D W_{m, n}(t, x) \cdot b(t, x, u, v)+f_{m, n}\left(t, x, W_{m, n}(t, x), D W_{m, n}(t, x) .\right. \\
\sigma(t, x, u, v), u, v)\}\}=0, \\
W_{m, n}(T, x)=\Phi(x), \quad x \in \mathbb{R}^{n},
\end{array}\right.
$$

where

$$
\begin{array}{r}
f_{m, n}(t, x, y, z, u, v)=f(t, x, y, z, u, v)-m\left(h^{\prime}(t, x)-y\right)^{-}+n(y-h(t, x))^{-}, \\
(t, x, y, z, u, v) \in[0, T] \times \mathbb{R}^{n} \times \mathbb{R} \times \mathbb{R}^{d} \times U \times V .
\end{array}
$$

Furthermore, from Theorem 4.2 in [4] we know, for any $m \in \mathbf{N}$, we have

$$
\lim _{n \rightarrow \infty} \uparrow W_{m, n}(t, x)=W_{m}(t, x) .
$$

Similarly, for any $m \in \mathbf{N}$, we have

$$
\lim _{n \rightarrow \infty} \downarrow W_{n, m}(t, x)=\bar{W}_{m}(t, x) .
$$

Theorem 4.3. $\lim _{m \rightarrow \infty} W_{m, m}(t, x)=W(t, x)$ for any $(t, x) \in[0, T] \times \mathbb{R}^{n}$.

Proof. From the comparison theorem for BSDEs (Lemma 2.2) and the definition of $W_{m, n}(t, x)$ we can get that for any $n \geq m, W_{n, m}(t, x) \leq W_{n, n}(t, x) \leq$ $W_{m, n}(t, x)$. Combining this with (4.15) and (4.16) we get

$$
\bar{W}_{m}(t, x) \leq \underline{\lim }_{n \rightarrow \infty} W_{n, n}(t, x) \leq \overline{\lim }_{n \rightarrow \infty} W_{n, n}(t, x) \leq W_{m}(t, x),
$$

and then taking the limit as $m \rightarrow \infty$ from Theorem 4.2 we have the wished result.

\section{Viscosity solution of Isaacs' equation with obstacles: uniqueness theorem}

The objective of this section is to study the uniqueness of the viscosity solution of Isaacs' equation (4.1),

$$
\left\{\begin{array}{l}
\min \left\{W(t, x)-h(t, x), \max \left[-\frac{\partial}{\partial t} W(t, x)-H^{-}(t, x, W, D W,\right.\right. \\
\left.\left.\left.D^{2} W\right), W(t, x)-h^{\prime}(t, x)\right]\right\}=0, \\
W(T, x)=\Phi(x) .
\end{array}\right.
$$


associated with the Hamiltonian

$$
\begin{aligned}
& H^{-}(t, x, y, q, X) \\
& \quad=\sup _{u \in U} \inf _{v \in V}\left\{\frac{1}{2} \operatorname{tr}\left(\sigma \sigma^{T}(t, x, u, v) X\right)+q . b(t, x, u, v)+f(t, x, y, q . \sigma, u, v)\right\},
\end{aligned}
$$

$t \in[0, T], x \in \mathbb{R}^{n}, y \in \mathbb{R}, q \in \mathbb{R}^{n}, X \in \mathbb{S}^{n}$. The functions $b, \sigma, f, h, h^{\prime}$ and $\Phi$ are still supposed to satisfy (H3.1) and (H3.2), respectively.

For the proof of the uniqueness of the viscosity solution for Eq. (5.1) in the space of functions

$$
\begin{aligned}
\Theta= & \left\{\varphi:[0, T] \times \mathbb{R}^{n} \rightarrow \mathbb{R} \mid \exists \widetilde{A}>0\right. \text { such that } \\
& \left.\lim _{|x| \rightarrow \infty} \varphi(t, x) \exp \left\{-\widetilde{A}\left[\log \left(\left(|x|^{2}+1\right)^{\frac{1}{2}}\right)\right]^{2}\right\}=0, \text { uniformly in } t \in[0, T]\right\}
\end{aligned}
$$

we borrow the main idea from Barles et al. [1]. This growth condition was introduced in [1] to prove the uniqueness of the viscosity solution of an integro-partial differential equation associated with a decoupled FBSDE with jumps. It was shown in [1] that this kind of growth condition is optimal for the uniqueness and can, in general, not be weakened, even not for PDEs. We adapt the ideas developed in $[1,3,4]$ to Isaacs' equation (5.1) to prove the uniqueness of the viscosity solution in $\Theta$. Since the proof of the uniqueness in $\Theta$ for Eq. (4.2) is the same we will restrict ourselves to that of (5.1). Before stating the main result of this section, let us begin with two auxiliary lemmata. Denoting by $K$ a Lipschitz constant of $f(t, x, .,$.$) , that is uniform in (t, x)$, we have the following

Lemma 5.1. Let an upper semicontinuous function $u_{1} \in \Theta$ be a viscosity subsolution and a lower semicontinuous function $u_{2} \in \Theta$ be a viscosity supersolution of Eq. (5.1). Then, the upper semicontinuous function $\omega:=u_{1}-u_{2}$ is a viscosity subsolution of the equation

$$
\left\{\begin{array}{l}
\min \left\{\omega(t, x),-\frac{\partial}{\partial t} \omega(t, x)-\sup _{u \in U, v \in V}\left(\frac{1}{2} \operatorname{tr}\left(\sigma \sigma^{T}(t, x, u, v) D^{2} \omega\right)+D \omega \cdot b(t, x, u, v)\right.\right. \\
\quad+K|\omega|+K|D \omega \cdot \sigma(t, x, u, v)|)\}=0,(t, x) \in[0, T) \times \mathbb{R}^{n} \\
\omega(T, x)=0, \quad x \in \mathbb{R}^{n} .
\end{array}\right.
$$

Proof. The proof is similar to that of Lemma 3.7 in [1] or Lemma 5.1 in [3], the main difference consists in the fact that here we have to deal with an obstacle problem.

We observe that $\omega(T, x)=u_{1}(T, x)-u_{2}(T, x) \leq \Phi(x)-\Phi(x)=0$, for all $x \in \mathbb{R}^{n}$. Let now $\left(t_{0}, x_{0}\right) \in[0, T) \times \mathbb{R}^{n}$ and $\varphi \in C^{3}\left([0, \bar{T}] \times \mathbb{R}^{n}\right)$ be such that $w-\varphi$ achieves a strict global maximum at $\left(t_{0}, x_{0}\right)$. For proving the theorem it suffices to show that

$$
\begin{aligned}
& \min \left\{\omega\left(t_{0}, x_{0}\right),-\frac{\partial}{\partial t} \varphi\left(t_{0}, x_{0}\right)-\sup _{u \in U, v \in V}\left(\frac { 1 } { 2 } \operatorname { t r } \left(\sigma \sigma^{T}\left(t_{0}, x_{0}, u, v\right) D^{2} \varphi\left(t_{0}, x_{0}\right)\right.\right.\right. \\
& \left.\left.\quad+D \varphi \cdot\left(t_{0}, x_{0}\right) b\left(t_{0}, x_{0}, x, u, v\right)+K\left|\varphi\left(t_{0}, x_{0}\right)\right|+K\left|D \varphi\left(t_{0}, x_{0}\right) \cdot \sigma\left(t_{0}, x_{0}, u, v\right)\right|\right)\right\} \leq 0 .
\end{aligned}
$$


For this end, applying the method of the separation of variables we introduce the function

$$
\Phi_{\varepsilon, \alpha}(t, x, s, y)=u_{1}(t, x)-u_{2}(s, y)-\frac{|x-y|^{2}}{\varepsilon^{2}}-\frac{(t-s)^{2}}{\alpha^{2}}-\varphi(t, x),
$$

where $\varepsilon$ and $\alpha$ are positive parameters which are devoted to tend to zero.

Since $\left(t_{0}, x_{0}\right)$ is a strict global maximum point of $w-\varphi$, there exists a sequence $(\bar{t}, \bar{x}, \bar{s}, \bar{y})$ such that

(i) $(\bar{t}, \bar{x}, \bar{s}, \bar{y})$ is a global maximum point of $\Phi_{\varepsilon, \alpha}$ in $[0, T] \times \bar{B}_{r} \times \bar{B}_{r}$ where $B_{r}$ is a ball with a large radius $r$;

(ii) $(\bar{t}, \bar{x}),(\bar{s}, \bar{y}) \rightarrow\left(t_{0}, x_{0}\right)$ as $(\varepsilon, \alpha) \rightarrow 0$;

(iii) $\frac{|\bar{x}-\bar{y}|^{2}}{\varepsilon^{2}}, \frac{(\bar{t}-\bar{s})^{2}}{\alpha^{2}}$ are bounded and tend to zero when $(\varepsilon, \alpha) \rightarrow 0$.

Since $u_{2}$ is lower semicontinuous we have $\underline{\lim }_{(\varepsilon, \alpha) \rightarrow 0} u_{2}(\bar{s}, \bar{y}) \geq u_{2}\left(t_{0}, x_{0}\right)$; and thanks to the upper semicontinuity of $u_{1}$ we have $\overline{\lim }_{(\varepsilon, \alpha) \rightarrow 0} u_{1}(\bar{t}, \bar{x}) \leq u_{1}\left(t_{0}, x_{0}\right)$. On the other hand, from $\Phi_{\varepsilon, \alpha}(\bar{t}, \bar{x}, \bar{s}, \bar{y}) \geq \Phi_{\varepsilon, \alpha}\left(t_{0}, x_{0}, t_{0}, x_{0}\right)$ we get

$u_{2}(\bar{s}, \bar{y}) \leq u_{1}(\bar{t}, \bar{x})-u_{1}\left(t_{0}, x_{0}\right)+u_{2}\left(t_{0}, x_{0}\right)+\varphi\left(t_{0}, x_{0}\right)-\varphi(\bar{t}, \bar{x})-\frac{|\bar{x}-\bar{y}|^{2}}{\varepsilon^{2}}-\frac{(\bar{t}-\bar{s})^{2}}{\alpha^{2}}$.

This yields $\varlimsup_{(\varepsilon, \alpha) \rightarrow 0} u_{2}(\bar{s}, \bar{y}) \leq u_{2}\left(t_{0}, x_{0}\right)$. Therefore, we have

(iv) $\lim _{(\varepsilon, \alpha) \rightarrow 0} u_{2}(\bar{s}, \bar{y})=u_{2}\left(t_{0}, x_{0}\right)$.

Analogously, we also get

(v) $\lim _{(\varepsilon, \alpha) \rightarrow 0} u_{1}(\bar{t}, \bar{x})=u_{1}\left(t_{0}, x_{0}\right)$.

Since $(\bar{t}, \bar{x}, \bar{s}, \bar{y})$ is a local maximum point of $\Phi_{\varepsilon, \alpha}, u_{2}(s, y)+\frac{|\bar{x}-y|^{2}}{\varepsilon^{2}}+\frac{(\bar{t}-s)^{2}}{\alpha^{2}}$ achieves in $(\bar{s}, \bar{y})$ a local minimum and from the definition of a viscosity supersolution of Eq. (4.1) we have $u_{2}(\bar{s}, \bar{y}) \geq h(\bar{s}, \bar{y})$. From (iv) we get $u_{2}\left(t_{0}, x_{0}\right) \geq h\left(t_{0}, x_{0}\right)$. If $\omega\left(t_{0}, x_{0}\right) \leq 0$, relation (5.3) is trivially fulfilled. So let us suppose that $\omega\left(t_{0}, x_{0}\right)>$ 0 . In this case, we have $h\left(t_{0}, x_{0}\right) \leq u_{2}\left(t_{0}, x_{0}\right)<u_{1}\left(t_{0}, x_{0}\right)$. Then according to $(\mathrm{v})$, and since $h$ is continuous we have

(vi) $u_{1}(\bar{t}, \bar{x})>h(\bar{t}, \bar{x})$, for $\varepsilon>0$ and $\alpha>0$ sufficiently small.

Similarly, since $u_{1}(t, x)-\frac{|x-\bar{y}|^{2}}{\varepsilon^{2}}-\frac{(t-\bar{s})^{2}}{\alpha^{2}}-\varphi(t, x)$ achieves in $(\bar{t}, \bar{x})$ a local maximum and from the definition of a viscosity subsolution of Eq. (4.1) and (vi) we have $u_{1}(\bar{t}, \bar{x}) \leq h^{\prime}(\bar{t}, \bar{x})$. From $(\mathrm{v})$ we get $u_{1}\left(t_{0}, x_{0}\right) \leq h^{\prime}\left(t_{0}, x_{0}\right)$. Therefore now we get $u_{2}\left(t_{0}, x_{0}\right)<h^{\prime}\left(t_{0}, x_{0}\right)$. Similarly, according to (iv) and $h^{\prime}$ is continuous we have

(vii) $u_{2}(\bar{s}, \bar{y})<h^{\prime}(\bar{s}, \bar{y})$, for $\varepsilon>0$ and $\alpha>0$ sufficiently small.

The properties (i) to (vii) and the fact that $u_{1}$ is a viscosity subsolution and $u_{2}$ a viscosity supersolution of Eq. (5.1) allow to proceed in the rest of the proof of this lemma exactly as in the proof of Lemma 3.7 in [1] (our situation here is even simpler because, contrary to Lemma 3.7 in [1], we don't have any integral 
part in Eq. (5.1)). So we get:

$$
\begin{aligned}
& -\frac{\partial \varphi}{\partial t}\left(t_{0}, x_{0}\right)-\sup _{u \in U, v \in V}\left\{\frac{1}{2} \operatorname{tr}\left(\left(\sigma \sigma^{T}\right)\left(t_{0}, x_{0}, u, v\right) D^{2} \varphi\left(t_{0}, x_{0}\right)\right)\right. \\
& \left.\quad+D \varphi\left(t_{0}, x_{0}\right) b\left(t_{0}, x_{0}, u, v\right)+K\left|\omega\left(t_{0}, x_{0}\right)\right|+K\left|D \varphi\left(t_{0}, x_{0}\right) \sigma\left(t_{0}, x_{0}, u, v\right)\right|\right\} \leq 0,
\end{aligned}
$$

from which relation (5.3) follows easily. Therefore $\omega$ is a viscosity subsolution of Eq. (5.2) and the proof is complete.

We now can establish the following comparison principle which is the key for the uniqueness for Eq. (5.1).

Theorem 5.1. We assume that (H3.1) and (H3.2) hold. Let an upper semicontinuous function $u_{1}$ (resp., a lower semicontinuous function $u_{2}$ ) $\in \Theta$ be a viscosity subsolution (resp., supersolution) of Eq. (5.1). Then we have

$$
u_{1}(t, x) \leq u_{2}(t, x), \quad \text { for all }(t, x) \in[0, T] \times \mathbb{R}^{n} .
$$

If, in particular, both $u_{1}$ and $u_{2}$ are continuous viscosity solutions from the class $\Theta$ then they coincide on $[0, T] \times \mathbb{R}^{n}$.

Proof. Theorem 5.1 in [4] establishes a comparison principle for Hamilton-JacobiBellman equations with obstable of type (5.2). Such an equation is related to controlled BSDE with one reflecting barrier, studied in that paper. Letting $\omega_{1}=$ $u_{1}-u_{2}$ we know from Lemma 5.1 that $\omega_{1}$ is a viscosity subsolution of Eq. (5.2). On the other hand, $\omega_{2}=0$ is, obviously, a viscosity solution and, hence, also a viscosity supersolution of Eq. (5.2). Both functions $\omega_{1}$ and $\omega_{2}$ are in $\Theta$, and the comparison principle stated in Theorem 5.1 of [4] yields that $u_{1}-u_{2}=\omega_{1} \leq \omega_{2}=0$, i.e., $u_{1} \leq u_{2}$ on $[0, T] \times R^{n}$. Finally, if $u_{1}, u_{2}$ are viscosity solutions of $(5.2)$, they are both viscosity sub- and supersolution, and from the just proved comparison result we get the equality of $u_{1}$ and $u_{2}$.

Remark 5.1. Obviously, since $\widetilde{W}(t, x)=\lim _{m \rightarrow \infty} \downarrow W_{m}(t, x)(\geq W(t, x))$, and $\widetilde{\bar{W}}(t, x)=\lim _{m \rightarrow \infty} \uparrow \bar{W}_{m}(t, x)(\leq W(t, x))$ (for their definitions, see Lemma 4.1 and Remarks 4.2 and 4.3), are a viscosity subsolution and a supersolution, respectively (see Proposition 4.1 and 4.2), and both are of linear growth, we have due to Theorem 5.1 that $\widetilde{W}(t, x)=W(t, x)=\widetilde{W}(t, x),(t, x) \in[0, T] \times \mathbb{R}^{n}$. Consequently, $W$ is a viscosity solution of (5.1), and it is unique in the class $\tilde{\Theta}$. Similarly we get that the upper value function $U(t, x)$ is the unique viscosity solution in $\tilde{\Theta}$ of Eq. (4.2).

Let us also remark that, since $H^{-} \leq H^{+}$on $[0, T] \times \mathbb{R}^{n}$, any viscosity solution of Eq. (4.2) is a supersolution of Eq. (5.1). Then, again from Theorem 5.1, it follows that $W \leq U$. This justifies calling $W$ lower value function and $U$ upper value function. 
Remark 5.2. If the Isaacs' condition holds, that is, if for all $(t, x, y, p, X) \in[0, T] \times$ $\mathbb{R}^{n} \times \mathbb{R} \times \mathbb{R}^{n} \times \mathbb{S}^{n}$,

$$
H^{-}(t, x, y, p, X)=H^{+}(t, x, y, p, X) \text {, }
$$

then the Eqs. (5.1) and (4.2) coincide, and from the uniqueness of the viscosity solution in $\Theta$ it follows that the lower value function $W(t, x)$ equals to the upper value function $U(t, x)$, that means, the associated stochastic differential game with reflection has a value.

\section{Appendix}

\subsection{RBSDES with two barriers associated with forward SDEs}

In this section we give an overview over basic results on RBSDEs with two barriers associated with Forward SDEs (for short: FSDEs). We consider measurable functions $b:[0, T] \times \Omega \times \mathbb{R}^{n} \rightarrow \mathbb{R}^{n}$ and $\sigma:[0, T] \times \Omega \times \mathbb{R}^{n} \rightarrow \mathbb{R}^{n \times d}$ which are supposed to satisfy the following conditions:

(i) $b(\cdot, x)$ and $\sigma(\cdot, x)$ are $\mathbb{F}$-adapted processes, and there exists some constant $C>0$ such that

$$
|b(t, x)|+|\sigma(t, x)| \leq C(1+|x|), a . s ., \text { for all } 0 \leq t \leq T, x \in \mathbb{R}^{n} ;
$$

(ii) $\quad b$ and $\sigma$ are Lipschitz in $x$, i.e., there is some constant $C>0$ such that

$$
\begin{aligned}
& \left|b(t, x)-b\left(t, x^{\prime}\right)\right|+\left|\sigma(t, x)-\sigma\left(t, x^{\prime}\right)\right| \leq C\left|x-x^{\prime}\right|, \text { a.s. }, \\
& \quad \text { for all } 0 \leq t \leq T, x, x^{\prime} \in \mathbb{R}^{n} .
\end{aligned}
$$

We now consider the following SDE parameterized by the initial condition $(t, \zeta) \in[0, T] \times L^{2}\left(\Omega, \mathcal{F}_{t}, P ; \mathbb{R}^{n}\right):$

$$
\left\{\begin{aligned}
d X_{s}^{t, \zeta} & =b\left(s, X_{s}^{t, \zeta}\right) d s+\sigma\left(s, X_{s}^{t, \zeta}\right) d B_{s}, s \in[t, T], \\
X_{t}^{t, \zeta} & =\zeta .
\end{aligned}\right.
$$

Under the assumption (H6.1), SDE (6.1) has a unique strong solution and, for any $p \geq 2$, there exists $C_{p} \in \mathbb{R}$ such that, for any $t \in[0, T]$ and $\zeta, \zeta^{\prime} \in L^{p}\left(\Omega, \mathcal{F}_{t}, P ; \mathbb{R}^{n}\right)$,

$$
\begin{array}{r}
E\left[\sup _{t \leq s \leq T}\left|X_{s}^{t, \zeta}-X_{s}^{t, \zeta^{\prime}}\right| p \mid \mathcal{F}_{t}\right] \leq C_{p}\left|\zeta-\zeta^{\prime}\right|^{p}, \quad \text { a.s. } \\
E\left[\sup _{t \leq s \leq T}\left|X_{s}^{t, \zeta}\right|^{p} \mid \mathcal{F}_{t}\right] \leq C_{p}\left(1+|\zeta|^{p}\right), \quad \text { a.s. }
\end{array}
$$

These well-known standard estimates can be consulted, for instance, in Ikeda, Watanabe [20], pp. 166-168, and also in Karatzas, Shreve [21], pp. 289-290. We emphasize that the constant $C_{p}$ in (6.2) only depends on the Lipschitz and the growth constants of $b$ and $\sigma$. 
Let now be given three real valued functions $f(t, x, y, z), \Phi(x)$ and $h(t, x)$, $h^{\prime}(t, x)$ which shall satisfy the following conditions:

(i) $\Phi: \Omega \times \mathbb{R}^{n} \rightarrow \mathbb{R}$ is an $\mathcal{F}_{T} \otimes \mathcal{B}\left(\mathbb{R}^{n}\right)$-measurable random variable and $f:[0, T] \times \Omega \times \mathbb{R}^{n} \times \mathbb{R} \times \mathbb{R}^{d} \rightarrow \mathbb{R}, \quad h, h^{\prime}: \Omega \times[0, T] \times \mathbb{R}^{n} \rightarrow \mathbb{R}$

are measurable processes such that,

$f(\cdot, x, y, z), h(\cdot, x), h^{\prime}(\cdot, x)$ are $\mathbb{F}$-adapted, for all $(x, y, z) \in \mathbb{R}^{n} \times \mathbb{R} \times \mathbb{R}^{d} ;$

(ii) There exists a constant $\mu>0$ such that, P-a.s.,

$\left|f(t, x, y, z)-f\left(t, x^{\prime}, y^{\prime}, z^{\prime}\right)\right| \leq \mu\left(\left|x-x^{\prime}\right|+\left|y-y^{\prime}\right|+\left|z-z^{\prime}\right|\right) ;$

$\left|\Phi(x)-\Phi\left(x^{\prime}\right)\right| \leq \mu\left|y-y^{\prime}\right|$

$\left|h(t, x)-h\left(t, x^{\prime}\right)\right|+\left|h^{\prime}(t, x)-h^{\prime}\left(t, x^{\prime}\right)\right| \leq \mu\left|x-x^{\prime}\right|$

for all $0 \leq t \leq T, x, x^{\prime} \in \mathbb{R}^{n}, y, y^{\prime} \in \mathbb{R}$ and $z, z^{\prime} \in \mathbb{R}^{d}$;

(iii) $f, \Phi$ and $h$ satisfy a linear growth condition, i.e., there exists some $C>0$ such that, $\mathrm{dt} \times \mathrm{dP}$-a.e., for all $x \in \mathbb{R}^{n}$,

and, moreover,

$$
|f(t, x, 0,0)|+|\Phi(x)|+|h(t, x)| \leq C(1+|x|)
$$

$$
\begin{aligned}
& h(\cdot, x), h^{\prime}(\cdot, x) \text { are continuous in } t, h(t, x)<h^{\prime}(t, x), \text { a.s., and } \\
& h(T, x) \leq \Phi(x) \leq h^{\prime}(T, x), \text { a.s., for all }(t, x) \in[0, T] \times \mathbb{R}^{n} .
\end{aligned}
$$

With the help of the above assumptions we can verify that the coefficient $f\left(s, X_{s}^{t, \zeta}, y, z\right)$ satisfies the hypotheses $(\mathrm{A} 1),(\mathrm{A} 2), \xi:=\Phi\left(X_{T}^{t, \zeta}\right) \in L^{2}\left(\Omega, \mathcal{F}_{T}, P ; \mathbb{R}\right)$ and $L_{s}:=h\left(s, X_{s}^{t, \zeta}\right), U_{s}:=h^{\prime}\left(s, X_{s}^{t, \zeta}\right) \in \mathcal{S}^{2}(0, T ; \mathbb{R})$. Therefore, according to Lemma 2.6 the following RBSDE with two barriers possesses a unique solution:

(i) $Y^{t, \zeta} \in \mathcal{S}^{2}(0, T ; \mathbb{R}), Z^{t, \zeta} \in \mathcal{H}\left(0, T ; \mathbb{R}^{d}\right)$ and $K^{+, t, \zeta}, K^{-, t, \zeta} \in \mathcal{A}_{c}(0, T ; \mathbb{R}) ;$

(ii) $Y_{s}^{t, \zeta}=\Phi\left(X_{T}^{t, \zeta}\right)+\int_{s}^{T} f\left(r, X_{r}^{t, \zeta}, Y_{r}^{t, \zeta}, Z_{r}^{t, \zeta}\right) d r+\left(K_{T}^{+, t, \zeta}-K_{s}^{+, t, \zeta}\right)-\left(K_{T}^{-, t, \zeta}\right.$ $\left.-K_{s}^{-, t, \zeta}\right)-\int_{s}^{T} Z_{r}^{t, \zeta} d B_{r}, s \in[t, T]$

(iii) $h\left(s, X_{s}^{t, \zeta}\right) \leq Y_{s}^{t, \zeta} \leq h^{\prime}\left(s, X_{s}^{t, \zeta}\right)$, a.s., for any $s \in[t, T]$;

(iv) $\int_{t}^{T}\left(Y_{r}^{t, \zeta}-h\left(r, X_{r}^{t, \zeta}\right)\right) d K_{r}^{+, t, \zeta}=\int_{t}^{T}\left(h^{\prime}\left(r, X_{r}^{t, \zeta}\right)-Y_{r}^{t, \zeta}\right) d K_{r}^{-, t, \zeta}=0$.

Proposition 6.1. We suppose that the hypotheses (H6.1) and (H6.2) hold. Then, for any $0 \leq t \leq T$ and the associated initial conditions $\zeta, \zeta^{\prime} \in L^{2}\left(\Omega, \mathcal{F}_{t}, P ; \mathbb{R}^{n}\right)$, we have the following estimates:

(i) $E\left[\sup _{t \leq s \leq T}\left|Y_{s}^{t, \zeta}\right|^{2} \mid \mathcal{F}_{t}\right] \leq C\left(1+|\zeta|^{2}\right)$, a.s.;

(ii) $E\left[\sup _{t \leq s \leq T}\left|Y_{s}^{t, \zeta}-Y_{s}^{t, \zeta^{\prime}}\right|^{2} \mid \mathcal{F}_{t}\right] \leq C\left|\zeta-\zeta^{\prime}\right|^{2}$, a.s.

In particular,

(iii) $\left|Y_{t}^{t, \zeta}\right| \leq C(1+|\zeta|)$, a.s.;

(iv) $\left|Y_{t}^{t, \zeta}-Y_{t}^{t, \zeta^{\prime}}\right| \leq C\left|\zeta-\zeta^{\prime}\right|$, a.s.

The above constant $C>0$ depends only on the Lipschitz and the growth constants of $b, \sigma, f, \Phi$ and $h$. 
Proof. From Lemma 2.8 combined with (6.2) we obtain easily (i). So we need only to prove (ii). For an arbitrarily fixed $\varepsilon>0$, we define the function $\psi_{\varepsilon}(x)=$ $\left(|x|^{2}+\varepsilon\right)^{\frac{1}{2}}, x \in \mathbb{R}^{n}$. Obviously, $|x| \leq \psi_{\varepsilon}(x) \leq|x|+\varepsilon^{\frac{1}{2}}, x \in \mathbb{R}^{n}$. Furthermore, for all $x \in \mathbb{R}^{n}$,

$$
D \psi_{\varepsilon}(x)=\frac{x}{\left(|x|^{2}+\varepsilon\right)^{\frac{1}{2}}}, \quad D^{2} \psi_{\varepsilon}(x)=\frac{I}{\left(|x|^{2}+\varepsilon\right)^{\frac{1}{2}}}-\frac{x \otimes x}{\left(|x|^{2}+\varepsilon\right)^{\frac{3}{2}}} .
$$

Then, we have

$$
\left|D \psi_{\varepsilon}(x)\right| \leq 1, \quad\left|D^{2} \psi_{\varepsilon}(x)\right||x| \leq \frac{C}{\left(|x|^{2}+\varepsilon\right)^{\frac{1}{2}}}|x| \leq C, \quad x \in \mathbb{R}^{n},
$$

for some constant $C$ that doesn't depend on $\varepsilon$. Let us denote by $X^{t, \zeta}$ and $X^{t, \zeta^{\prime}}$ the unique solution of $\operatorname{SDE}(6.1)$ with initial data $(t, \zeta)$ and $\left(t, \zeta^{\prime}\right)$, respectively. Moreover, recall that $\mu$ is the Lipschitz constant of $h, h^{\prime}, \Phi, f$. We consider the following two RBSDEs:

(i) $\widetilde{Y} \in \mathcal{S}^{2}(0, T ; \mathbb{R}), \widetilde{Z} \in \mathcal{H}\left(0, T ; \mathbb{R}^{d}\right)$ and $\widetilde{K}^{+}, \widetilde{K}^{-} \in \mathcal{A}_{c}(0, T ; \mathbb{R})$;

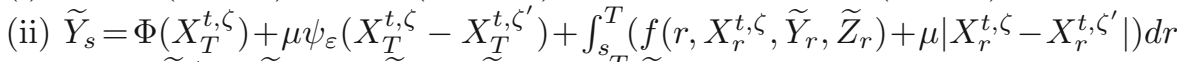
$+\left(\widetilde{K}_{T}^{+}-\widetilde{K}_{s}^{+}\right)-\left(\widetilde{K}_{T}^{-}-\widetilde{K}_{s}^{-}\right)-\int_{s}^{T} \widetilde{Z}_{r} d B_{r}, s \in[t, T] ;$

(iii) $h\left(s, X_{s}^{t, \zeta}\right)+\mu \psi_{\varepsilon}\left(X_{s}^{t, \zeta}-X_{s}^{t, \zeta^{\prime}}\right) \leq \tilde{Y}_{s} \leq h^{\prime}\left(s, X_{s}^{t, \zeta}\right)+\mu \psi_{\varepsilon}\left(X_{s}^{t, \zeta}-X_{s}^{t, \zeta^{\prime}}\right)$,

$$
\text { a.s., } s \in[t, T] \text {; }
$$

(iv) $\int_{t}^{T}\left(\widetilde{Y}_{r}-h\left(r, X_{r}^{t, \zeta}\right)-\mu \psi_{\varepsilon}\left(X_{r}^{t, \zeta}-X_{r}^{t, \zeta^{\prime}}\right)\right) d \widetilde{K}_{r}^{+}=\int_{t}^{T}\left(h^{\prime}\left(r, X_{r}^{t, \zeta}\right)\right.$ $\left.+\mu \psi_{\varepsilon}\left(X_{r}^{t, \zeta}-X_{r}^{t, \zeta^{\prime}}\right)-\widetilde{Y}_{r}\right) d \widetilde{K}_{r}^{-}=0$.

and

(i) $\bar{Y} \in \mathcal{S}^{2}(0, T ; \mathbb{R}), \bar{Z} \in \mathcal{H}\left(0, T ; \mathbb{R}^{d}\right)$ and $\bar{K}^{+}, \bar{K}^{-} \in \mathcal{A}_{c}(0, T ; \mathbb{R})$;

(ii) $\bar{Y}_{s}=\Phi\left(X_{T}^{t, \zeta}\right)-\mu \psi_{\varepsilon}\left(X_{T}^{t, \zeta}-X_{T}^{t, \zeta^{\prime}}\right)+\int_{s}^{T}\left(f\left(r, X_{r}^{t, \zeta}, \bar{Y}_{r}, \bar{Z}_{r}\right)-\mu\left|X_{r}^{t, \zeta}-X_{r}^{t, \zeta^{\prime}}\right|\right) d r$

$$
+\left(\bar{K}_{T}^{+}-\bar{K}_{s}^{+}\right)-\left(\bar{K}_{T}^{-}-\bar{K}_{s}^{-}\right)-\int_{s}^{T} \bar{Z}_{r} d B_{r}, \quad s \in[t, T]
$$

(iii) $h\left(s, X_{s}^{t, \zeta}\right)-\mu \psi_{\varepsilon}\left(X_{s}^{t, \zeta}-X_{s}^{t, \zeta^{\prime}}\right) \leq \bar{Y}_{s} \leq h^{\prime}\left(s, X_{s}^{t, \zeta}\right)-\mu \psi_{\varepsilon}\left(X_{s}^{t, \zeta}-X_{s}^{t, \zeta^{\prime}}\right)$,

$$
\text { a.s., } s \in[t, T] \text {; }
$$

(iv) $\int_{t}^{T}\left(\bar{Y}_{r}-h\left(r, X_{r}^{t, \zeta}\right)+\mu \psi_{\varepsilon}\left(X_{r}^{t, \zeta}-X_{r}^{t, \zeta^{\prime}}\right)\right) d \bar{K}_{r}^{+}=\int_{t}^{T}\left(h^{\prime}\left(r, X_{r}^{t, \zeta}\right)\right.$

$$
\left.-\mu \psi_{\varepsilon}\left(X_{r}^{t, \zeta}-X_{r}^{t, \zeta^{\prime}}\right)-\bar{Y}_{r}\right) d \bar{K}_{r}^{-}=0 \text {. }
$$

Obviously, their coefficients satisfy the assumptions in (H6.2) and they admit unique solutions $\left(\widetilde{Y}, \widetilde{Z}, \widetilde{K}^{+}, \widetilde{K}^{-}\right)$and $\left(\bar{Y}, \bar{Z}, \bar{K}^{+}, \bar{K}^{-}\right)$, respectively. Moreover, from the comparison theorem for RBSDEs with two barriers (Lemma 2.7)

$$
\bar{Y}_{s} \leq Y_{s}^{t, \zeta} \leq \widetilde{Y}_{s}, \quad \bar{Y}_{s} \leq Y_{s}^{t, \zeta^{\prime}} \leq \widetilde{Y}_{s}, \quad \text { P-a.s., for all } s \in[t, T]
$$


We shall still introduce two other RBSDEs with two reflecting barriers:

(i) $\widetilde{Y}^{\prime} \in \mathcal{S}^{2}(0, T ; \mathbb{R}), \widetilde{Z}^{\prime} \in \mathcal{H}\left(0, T ; \mathbb{R}^{d}\right)$ and $\widetilde{K}^{+^{\prime}}, \widetilde{K}^{-^{\prime}} \in \mathcal{A}_{c}(0, T ; \mathbb{R})$;

(ii) $\tilde{Y}_{s}^{\prime}=\Phi\left(X_{T}^{t, \zeta}\right)$

$$
\begin{aligned}
& +\int_{s}^{T}\left[f \left(r, X_{r}^{t, \zeta}, \widetilde{Y}_{r}^{\prime}+\mu \psi_{\varepsilon}\left(X_{r}^{t, \zeta}-X_{r}^{t, \zeta^{\prime}}\right), \widetilde{Z}_{r}^{\prime}+\mu D \psi_{\varepsilon}\left(X_{r}^{t, \zeta}-X_{r}^{t, \zeta^{\prime}}\right)\left(\sigma\left(r, X_{r}^{t, \zeta}\right)\right.\right.\right. \\
& \left.\left.\quad-\sigma\left(r, X_{r}^{t, \zeta^{\prime}}\right)\right)\right)+\mu\left|X_{r}^{t, \zeta}-X_{r}^{t, \zeta^{\prime}}\right|+\mu D \psi_{\varepsilon}\left(X_{r}^{t, \zeta}-X_{r}^{t, \zeta^{\prime}}\right)\left(b\left(r, X_{r}^{t, \zeta}\right)-b\left(r, X_{r}^{t, \zeta^{\prime}}\right)\right) \\
& +\frac{1}{2} \mu\left(D^{2} \psi_{\varepsilon}\left(X_{r}^{t, \zeta}-X_{r}^{t, \zeta^{\prime}}\right)\left(\sigma\left(r, X_{r}^{t, \zeta}\right)-\sigma\left(r, X_{r}^{t, \zeta^{\prime}}\right)\right), \sigma\left(r, X_{r}^{t, \zeta}\right)\right. \\
& \left.\left.\quad-\sigma\left(r, X_{r}^{t, \zeta^{\prime}}\right)\right)\right] d r+\left(\widetilde{K}_{T}^{t^{\prime}}-\widetilde{K}_{s}^{t^{\prime}}\right)-\left(\widetilde{K}_{T}^{-\prime}-\widetilde{K}_{s}^{-^{\prime}}\right)-\int_{s}^{T} \widetilde{Z}_{r}^{\prime} d B_{r}, \quad s \in[t, T] ;
\end{aligned}
$$

(iii) $h\left(s, X_{s}^{t, \zeta}\right) \leq \widetilde{Y}_{s}^{\prime} \leq h^{\prime}\left(s, X_{s}^{t, \zeta}\right)$, a.s., $s \in[t, T]$;

(iv) $\int_{t}^{T}\left(\widetilde{Y}_{r}^{\prime}-h\left(r, X_{r}^{t, \zeta}\right)\right) d \widetilde{K}_{r}^{+^{\prime}}=\int_{t}^{T}\left(h^{\prime}\left(r, X_{r}^{t, \zeta}\right)-\widetilde{Y}_{r}^{\prime}\right) d \widetilde{K}_{r}^{-{ }^{\prime}}=0$,

and

(i) $\bar{Y}^{\prime} \in \mathcal{S}^{2}(0, T ; \mathbb{R}), \bar{Z}^{\prime} \in \mathcal{H}\left(0, T ; \mathbb{R}^{d}\right)$ and $\bar{K}^{+^{\prime}}, \bar{K}^{{ }^{\prime}} \in \mathcal{A}_{c}(0, T ; \mathbb{R})$;

(ii) $\bar{Y}_{s}^{\prime}=\Phi\left(X_{T}^{t, \zeta}\right)+\int_{s}^{T}\left[f\left(r, X_{r}^{t, \zeta}, \bar{Y}_{r}^{\prime}-\mu \psi_{\varepsilon}\left(X_{r}^{t, \zeta}-X_{r}^{t, \zeta^{\prime}}\right), \bar{Z}_{r}^{\prime}\right.\right.$

$$
\begin{aligned}
& \left.-\mu D \psi_{\varepsilon}\left(X_{r}^{t, \zeta}-X_{r}^{t, \zeta^{\prime}}\right)\left(\sigma\left(r, X_{r}^{t, \zeta}\right)-\sigma\left(r, X_{r}^{t, \zeta^{\prime}}\right)\right)\right)-\mu\left|X_{r}^{t, \zeta}-X_{r}^{t, \zeta^{\prime}}\right| \\
& -\mu D \psi_{\varepsilon}\left(X_{r}^{t, \zeta}-X_{r}^{t, \zeta^{\prime}}\right)\left(b\left(r, X_{r}^{t, \zeta}\right)-b\left(r, X_{r}^{t, \zeta^{\prime}}\right)\right) \\
& -\frac{1}{2} \mu\left(D^{2} \psi_{\varepsilon}\left(X_{r}^{t, \zeta}-X_{r}^{t, \zeta^{\prime}}\right)\left(\sigma\left(r, X_{r}^{t, \zeta}\right)-\sigma\left(r, X_{r}^{t, \zeta^{\prime}}\right)\right), \sigma\left(r, X_{r}^{t, \zeta}\right)\right. \\
& \left.\left.-\sigma\left(r, X_{r}^{t, \zeta^{\prime}}\right)\right)\right] d r+\left(\bar{K}_{T}^{+^{\prime}}-\bar{K}_{s}^{+^{\prime}}\right)-\left(\bar{K}_{T}^{-^{\prime}}-\bar{K}_{s}^{-^{\prime}}\right)-\int_{s}^{T} \bar{Z}_{r}^{\prime} d B_{r}, \quad s \in[t, T] ;
\end{aligned}
$$

(iii) $h\left(s, X_{s}^{t, \zeta}\right) \leq \bar{Y}_{s}^{\prime} \leq h^{\prime}\left(s, X_{s}^{t, \zeta}\right)$, a.s., $s \in[t, T]$;

(iv) $\int_{t}^{T}\left(\bar{Y}_{r}^{\prime}-h\left(r, X_{r}^{t, \zeta}\right)\right) d \bar{K}_{r}^{+^{\prime}}=\int_{t}^{T}\left(h^{\prime}\left(r, X_{r}^{t, \zeta}\right)-\bar{Y}_{r}^{\prime}\right) d \bar{K}_{r}^{-^{\prime}}=0$.

Obviously, also the RBSDEs with two barriers (6.9) and (6.10) satisfy the assumption (H6.2) and, thus, admit unique solutions $\left(\widetilde{Y}^{\prime}, \widetilde{Z}^{\prime}, \widetilde{K}^{+^{\prime}}, \widetilde{K}^{-^{\prime}}\right)$ and $\left(\bar{Y}^{\prime}, \bar{Z}^{\prime}, \bar{K}^{+^{\prime}}, \bar{K}^{-^{\prime}}\right)$, respectively. On the other hand, from the uniqueness of the solution of RBSDE with two barriers we know that

$$
\begin{aligned}
& \widetilde{Y}_{s}^{\prime}=\widetilde{Y}_{s}-\mu \psi_{\varepsilon}\left(X_{s}^{t, \zeta}-X_{s}^{t, \zeta^{\prime}}\right), \text { for all } s \in[t, T], \text { P-a.s., } \\
& \widetilde{Z}_{s}^{\prime}=\widetilde{Z}_{s}-\mu D \psi_{\varepsilon}\left(X_{s}^{t, \zeta}-X_{s}^{t, \zeta^{\prime}}\right)\left(\sigma\left(s, X_{s}^{t, \zeta}\right)-\sigma\left(s, X_{s}^{t, \zeta^{\prime}}\right)\right), \operatorname{dsdP} \text {-a.e. on }[t, T] \times \Omega, \\
& \widetilde{K}_{s}^{+^{\prime}}=\widetilde{K}_{s}^{+}, \widetilde{K}_{s}^{{ }^{\prime}}=\widetilde{K}_{s}^{-} \text {for all } s \in[t, T], \text { P-a.s. }
\end{aligned}
$$

and

$\bar{Y}_{s}^{\prime}=\bar{Y}_{s}+\mu \psi_{\varepsilon}\left(X_{s}^{t, \zeta}-X_{s}^{t, \zeta^{\prime}}\right)$, for all $s \in[t, T]$, P-a.s.,

$\bar{Z}_{s}^{\prime}=\bar{Z}_{s}+\mu D \psi_{\varepsilon}\left(X_{s}^{t, \zeta}-X_{s}^{t, \zeta^{\prime}}\right)\left(\sigma\left(s, X_{s}^{t, \zeta}\right)-\sigma\left(s, X_{s}^{t, \zeta^{\prime}}\right)\right)$, dsdP-a.e. on $[t, T] \times \Omega$, $\bar{K}_{s}^{+^{\prime}}=\bar{K}_{s}^{+}, \bar{K}_{s}^{-^{\prime}}=\bar{K}_{s}^{-}$, for all $s \in[t, T]$, P-a.s. 
Then, for the notations introduced in Lemma 2.9 we have

$$
\begin{aligned}
& \Delta g\left(r, \widetilde{Y}_{r}^{\prime}, \widetilde{Z}_{r}^{\prime}\right) \\
& =f\left(r, X_{r}^{t, \zeta}, \widetilde{Y}_{r}^{\prime}+\mu \psi_{\varepsilon}\left(X_{r}^{t, \zeta}-X_{r}^{t, \zeta^{\prime}}\right), \widetilde{Z}_{r}^{\prime}+\mu D \psi_{\varepsilon}\left(X_{r}^{t, \zeta}-X_{r}^{t, \zeta^{\prime}}\right)\left(\sigma\left(r, X_{r}^{t, \zeta}\right)\right.\right. \\
& \left.\left.-\sigma\left(r, X_{r}^{t, \zeta^{\prime}}\right)\right)\right)-f\left(r, X_{r}^{t, \zeta}, \tilde{Y}_{r}^{\prime}-\mu \psi_{\varepsilon}\left(X_{r}^{t, \zeta}-X_{r}^{t, \zeta^{\prime}}\right), \widetilde{Z}_{r}^{\prime}\right. \\
& \left.-\mu D \psi_{\varepsilon}\left(X_{r}^{t, \zeta}-X_{r}^{t, \zeta^{\prime}}\right)\left(\sigma\left(r, X_{r}^{t, \zeta}\right)-\sigma\left(r, X_{r}^{t, \zeta^{\prime}}\right)\right)\right)+2 \mu\left|X_{r}^{t, \zeta}-X_{r}^{t, \zeta^{\prime}}\right| \\
& +2 \mu D \psi_{\varepsilon}\left(X_{r}^{t, \zeta}-X_{r}^{t, \zeta^{\prime}}\right)\left(b\left(r, X_{r}^{t, \zeta}\right)-b\left(r, X_{r}^{t, \zeta^{\prime}}\right)\right) \\
& +\mu\left(D^{2} \psi_{\varepsilon}\left(X_{r}^{t, \zeta}-X_{r}^{t, \zeta^{\prime}}\right)\left(\sigma\left(r, X_{r}^{t, \zeta}\right)-\sigma\left(r, X_{r}^{t, \zeta^{\prime}}\right)\right), \sigma\left(r, X_{r}^{t, \zeta}\right)-\sigma\left(r, X_{r}^{t, \zeta^{\prime}}\right)\right) ; \\
& \Delta \xi=0 .
\end{aligned}
$$

From (6.5) and the Lipschitz continuity of $f, b$ and $\sigma$ we get

$$
\left|\Delta g\left(r, \widetilde{Y}_{r}^{\prime}, \widetilde{Z}_{r}^{\prime}\right)\right| \leq C\left|X_{r}^{t, \zeta}-X_{r}^{t, \zeta^{\prime}}\right|+C \varepsilon^{\frac{1}{2}}, \text { P-a.s. }
$$

where the constant $C$ is independent of $\varepsilon$. Therefore, from Lemma 2.9 and (6.2) we get

$$
E\left[\sup _{t \leq s \leq T}\left|\tilde{Y}_{s}^{\prime}-\bar{Y}_{s}^{\prime}\right|^{2} \mid \mathcal{F}_{t}\right] \leq C\left|\zeta-\zeta^{\prime}\right|^{2}+C \varepsilon, \text { P-a.s. }
$$

Furthermore, from (6.8), (6.11), (6.12) and (6.2) we have

$$
\begin{aligned}
& E\left[\sup _{t \leq s \leq T}\left|Y_{s}^{t, \zeta}-Y_{s}^{t, \zeta^{\prime}}\right|^{2} \mid \mathcal{F}_{t}\right] \leq E\left[\sup _{t \leq s \leq T}\left|\widetilde{Y}_{s}-\bar{Y}_{s}\right|^{2} \mid \mathcal{F}_{t}\right] \\
& \quad \leq 2 E\left[\sup _{t \leq s \leq T}\left|\widetilde{Y}_{s}^{\prime}-\bar{Y}_{s}^{\prime}\right|^{2} \mid \mathcal{F}_{t}\right]+16 \mu^{2}\left(E\left[\sup _{t \leq s \leq T}\left|X_{s}^{t, \zeta}-X_{s}^{t, \zeta^{\prime}}\right|^{2} \mid \mathcal{F}_{t}\right]+\varepsilon\right) \\
& \quad \leq C\left|\zeta-\zeta^{\prime}\right|^{2}+C \varepsilon, \text { P-a.s. }
\end{aligned}
$$

Finally, we let $\varepsilon$ tend to 0 , and we get (ii). The proof is complete.

Let us now introduce the random field:

$$
u(t, x)=\left.Y_{s}^{t, x}\right|_{s=t}, \quad(t, x) \in[0, T] \times \mathbb{R}^{n},
$$

where $Y^{t, x}$ is the solution of the RBSDE with two barriers (6.3), with $\zeta \in$ $L^{2}\left(\Omega, \mathcal{F}_{t}, P ; \mathbb{R}^{n}\right)$ being replaced by $x \in \mathbb{R}^{n}$.

As a consequence of Proposition 6.1 we have that, for all $t \in[0, T]$, P-a.s.,

(i) $|u(t, x)-u(t, y)| \leq C|x-y|$, for all $x, y \in \mathbb{R}^{n}$;

(ii) $|u(t, x)| \leq C(1+|x|)$, for all $x \in \mathbb{R}^{n}$.

The random field $u$ and $Y^{t, \zeta},(t, \zeta) \in[0, T] \times L^{2}\left(\Omega, \mathcal{F}_{t}, P ; \mathbb{R}^{n}\right)$, are related by the following theorem.

Proposition 6.2. Under the assumptions (H6.1) and (H6.2) we have, for any $t \in$ $[0, T]$ and $\zeta \in L^{2}\left(\Omega, \mathcal{F}_{t}, P ; \mathbb{R}^{n}\right)$,

$$
u(t, \zeta)=Y_{t}^{t, \zeta}, \quad P-a . s .
$$

The proof of Proposition 6.2 can be got by adapting the corresponding argument of Peng [25] to RBSDEs, we give it for the reader's convenience. It makes use of the following definition.

Defnition 6.1. For any $\mathrm{t} \in[0, T]$, a sequence $\left\{A_{i}\right\}_{i=1}^{N} \subset \mathcal{F}_{t}$ (with $1 \leq N \leq \infty$ ) is called a partition of $\left(\Omega, \mathcal{F}_{t}\right)$ if $\cup_{i=1}^{N} A_{i}=\Omega$ and $A_{i} \cap A_{j}=\phi$, whenever $i \neq j$. 
Proof (of Proposition 6.2). We first consider the case that $\zeta$ is a simple random variable of the form

$$
\zeta=\sum_{i=1}^{N} x_{i} \mathbf{1}_{A_{i}}
$$

where $\left\{A_{i}\right\}_{i=1}^{N}$ is a finite partition of $\left(\Omega, \mathcal{F}_{t}\right)$ and $x_{i} \in \mathbb{R}^{n}$, for $1 \leq i \leq N$. For each $i$, we put $\left(X_{s}^{i}, Y_{s}^{i}, Z_{s}^{i}\right) \equiv\left(X_{s}^{t, x_{i}}, Y_{s}^{t, x_{i}}, Z_{s}^{t, x_{i}}\right)$. Then $X^{i}$ is the solution of the SDE

$$
X_{s}^{i}=x_{i}+\int_{t}^{s} b\left(r, X_{r}^{i}\right) d r+\int_{t}^{s} \sigma\left(r, X_{r}^{i}\right) d B_{r}, s \in[t, T]
$$

and $\left(Y^{i}, Z^{i}, K^{+, i}, K^{-, i}\right)$ is the solution of the associated RBSDE

$$
\begin{aligned}
Y_{s}^{i}= & \Phi\left(X_{T}^{i}\right)+\int_{s}^{T} f\left(r, X_{r}^{i}, Y_{r}^{i}, Z_{r}^{i}\right) d r+\left(K_{T}^{+, i}-K_{s}^{+, i}\right)-\left(K_{T}^{-, i}-K_{s}^{-, i}\right) \\
& -\int_{s}^{T} Z_{r}^{i} d B_{r}, s \in[t, T], h\left(s, X_{s}^{i}\right) \leq Y_{s}^{i} \leq h^{\prime}\left(s, X_{s}^{i}\right), \quad \int_{t}^{T}\left(Y_{r}^{i}-h\left(r, X_{r}^{i}\right)\right) d K_{r}^{+, i} \\
= & \int_{t}^{T}\left(h^{\prime}\left(r, X_{r}^{i}\right)-Y_{r}^{i}\right) d K_{r}^{-, i}=0 .
\end{aligned}
$$

The above two equations are multiplied by $\mathbf{1}_{A_{i}}$ and summed up with respect to $i$. Thus, taking into account that $\sum_{i} \varphi\left(x_{i}\right) \mathbf{1}_{A_{i}}=\varphi\left(\sum_{i} x_{i} \mathbf{1}_{A_{i}}\right)$, for any function $\varphi$, we get

$$
\sum_{i=1}^{N} \mathbf{1}_{A_{i}} X_{s}^{i}=\sum_{i=1}^{N} x_{i} \mathbf{1}_{A_{i}}+\int_{t}^{s} b\left(r, \sum_{i=1}^{N} \mathbf{1}_{A_{i}} X_{r}^{i}\right) d r+\int_{t}^{s} \sigma\left(r, \sum_{i=1}^{N} \mathbf{1}_{A_{i}} X_{r}^{i}\right) d B_{r}
$$

and

$$
\begin{aligned}
& \sum_{i=1}^{N} \mathbf{1}_{A_{i}} Y_{s}^{i}=\Phi\left(\sum_{i=1}^{N} \mathbf{1}_{A_{i}} X_{T}^{i}\right)+\int_{s}^{T} f\left(r, \sum_{i=1}^{N} \mathbf{1}_{A_{i}} X_{r}^{i}, \sum_{i=1}^{N} \mathbf{1}_{A_{i}} Y_{r}^{i}, \sum_{i=1}^{N} \mathbf{1}_{A_{i}} Z_{r}^{i}\right) d r \\
& +\left(\sum_{i=1}^{N} \mathbf{1}_{A_{i}} K_{T}^{+, i}-\sum_{i=1}^{N} \mathbf{1}_{A_{i}} K_{s}^{+, i}\right)-\left(\sum_{i=1}^{N} \mathbf{1}_{A_{i}} K_{T}^{-, i}-\sum_{i=1}^{N} \mathbf{1}_{A_{i}} K_{s}^{-, i}\right) \\
& \quad-\int_{s}^{T} \sum_{i=1}^{N} \mathbf{1}_{A_{i}} Z_{r}^{i} d B_{r}, h\left(s, \sum_{i=1}^{N} \mathbf{1}_{A_{i}} X_{s}^{i}\right) \leq \sum_{i=1}^{N} \mathbf{1}_{A_{i}} Y_{s}^{i} \leq h^{\prime}\left(s, \sum_{i=1}^{N} \mathbf{1}_{A_{i}} X_{s}^{i}\right), \\
& \int_{t}^{T}\left(\sum_{i=1}^{N} \mathbf{1}_{A_{i}} Y_{r}^{i}-h\left(r, \sum_{i=1}^{N} \mathbf{1}_{A_{i}} X_{r}^{i}\right)\right) d\left(\sum_{i=1}^{N} \mathbf{1}_{A_{i}} K_{r}^{+, i}\right) \\
& \quad \int_{t}^{T}\left(h^{\prime}\left(r, \sum_{i=1}^{N} \mathbf{1}_{A_{i}} X_{r}^{i}\right)-\sum_{i=1}^{N} \mathbf{1}_{A_{i}} Y_{r}^{i}\right) d\left(\sum_{i=1}^{N} \mathbf{1}_{A_{i}} K_{r}^{-, i}\right)=0 .
\end{aligned}
$$


Then the strong uniqueness property of the SDE and the associated RBSDE with two barriers yields: For $s \in[t, T]$,

$$
\begin{aligned}
X_{s}^{t, \zeta} & =\sum_{i=1}^{N} X_{s}^{i} \mathbf{1}_{A_{i}},\left(Y_{s}^{t, \zeta}, Z_{s}^{t, \zeta}, K_{s}^{+, t, \zeta}, K_{s}^{-, t, \zeta}\right) \\
& =\left(\sum_{i=1}^{N} \mathbf{1}_{A_{i}} Y_{s}^{i}, \sum_{i=1}^{N} \mathbf{1}_{A_{i}} Z_{s}^{i}, \sum_{i=1}^{N} \mathbf{1}_{A_{i}} K_{s}^{+, i}, \sum_{i=1}^{N} \mathbf{1}_{A_{i}} K_{s}^{-, i}\right) .
\end{aligned}
$$

Finally, from $u\left(t, x_{i}\right)=Y_{t}^{i}, 1 \leq i \leq N$, we deduce that

$$
Y_{t}^{t, \zeta}=\sum_{i=1}^{N} Y_{t}^{i} \mathbf{1}_{A_{i}}=\sum_{i=1}^{N} u\left(t, x_{i}\right) \mathbf{1}_{A_{i}}=u\left(t, \sum_{i=1}^{N} x_{i} \mathbf{1}_{A_{i}}\right)=u(t, \zeta) .
$$

Therefore, for simple random variables, we have the desired result.

Given a general $\zeta \in L^{2}\left(\Omega, \mathcal{F}_{t}, P ; \mathbb{R}^{n}\right)$ we can choose a sequence of simple random variables $\left\{\zeta_{i}\right\}$ which converges to $\zeta$ in $L^{2}\left(\Omega, \mathcal{F}_{t}, P ; \mathbb{R}^{n}\right)$. Consequently, from the estimates $(6.4),(6.15)$ and the first step of the proof, we have

$$
\begin{aligned}
E\left|Y_{t}^{t, \zeta_{i}}-Y_{t}^{t, \zeta}\right|^{2} & \leq C E\left|\zeta_{i}-\zeta\right|^{2} \rightarrow 0, \quad i \rightarrow \infty, \\
E\left|u\left(t, \zeta_{i}\right)-u(t, \zeta)\right|^{2} & \leq C E\left|\zeta_{i}-\zeta\right|^{2} \rightarrow 0, \quad i \rightarrow \infty, \\
\text { and } \quad Y_{t}^{t, \zeta_{i}} & =u\left(t, \zeta_{i}\right), \quad i \geq 1 .
\end{aligned}
$$

Then the proof is complete.

\subsection{The proof of Theorem 3.1}

Proof. To simplify notations we put

$$
W_{\delta}(t, x)=\operatorname{essinf}_{\beta \in \mathcal{B}_{t, t+\delta}} \operatorname{esssup}_{u \in \mathcal{U}_{t, t+\delta}} G_{t, t+\delta}^{t, x ; u, \beta(u)}\left[W\left(t+\delta, X_{t+\delta}^{t, x ; u, \beta(u)}\right)\right] .
$$

The proof that $W_{\delta}(t, x)$ coincides with $W(t, x)$ will be split into a sequel of lemmata which all suppose that (H3.1) and (H3.2) are satisfied. Let us fix $(t, x) \in$ $[0, T] \times \mathbb{R}^{n}$.

Lemma 6.1. $W_{\delta}(t, x)$ is deterministic.

The proof of this lemma uses the same ideas as that of Proposition 3.1 so that it can be omitted here.

Lemma 6.2. $W_{\delta}(t, x) \leq W(t, x)$.

Proof. Let $\beta \in \mathcal{B}_{t, T}$ be arbitrarily fixed. Then, given a $u_{2}(\cdot) \in \mathcal{U}_{t+\delta, T}$, we define as follows the restriction $\beta_{1}$ of $\beta$ to $\mathcal{U}_{t, t+\delta}$ :

$$
\beta_{1}\left(u_{1}\right):=\left.\beta\left(u_{1} \oplus u_{2}\right)\right|_{[t, t+\delta]}, \quad u_{1}(\cdot) \in \mathcal{U}_{t, t+\delta},
$$

where $u_{1} \oplus u_{2}:=u_{1} \mathbf{1}_{[t, t+\delta]}+u_{2} \mathbf{1}_{(t+\delta, T]}$ extends $u_{1}(\cdot)$ to an element of $\mathcal{U}_{t, T}$. It is easy to check that $\beta_{1} \in \mathcal{B}_{t, t+\delta}$. Moreover, from the nonanticipativity property of $\beta$ we deduce that $\beta_{1}$ is independent of the special choice of $u_{2}(\cdot) \in \mathcal{U}_{t+\delta, T}$. Consequently, from the definition of $W_{\delta}(t, x)$,

$$
W_{\delta}(t, x) \leq \operatorname{esssup}_{u_{1} \in \mathcal{U}_{t, t+\delta}} G_{t, t+\delta}^{t, x ; u_{1}, \beta_{1}\left(u_{1}\right)}\left[W\left(t+\delta, X_{t+\delta}^{t, x ; u_{1}, \beta_{1}\left(u_{1}\right)}\right)\right], \text { P-a.s. }
$$


We use the notation $I_{\delta}(t, x, u, v):=G_{t, t+\delta}^{t, x ; u, v}\left[W\left(t+\delta, X_{t+\delta}^{t, x ; u, v}\right)\right]$ and notice that there exists a sequence $\left\{u_{i}^{1}, i \geq 1\right\} \subset \mathcal{U}_{t, t+\delta}$ such that

$I_{\delta}\left(t, x, \beta_{1}\right):=\operatorname{esssup}_{u_{1} \in \mathcal{U}_{t, t+\delta}} I_{\delta}\left(t, x, u_{1}, \beta_{1}\left(u_{1}\right)\right)=\sup _{i \geq 1} I_{\delta}\left(t, x, u_{i}^{1}, \beta_{1}\left(u_{i}^{1}\right)\right)$, P-a.s.

For any $\varepsilon>0$, we put $\widetilde{\Gamma}_{i}:=\left\{I_{\delta}\left(t, x, \beta_{1}\right) \leq I_{\delta}\left(t, x, u_{i}^{1}, \beta_{1}\left(u_{i}^{1}\right)\right)+\varepsilon\right\} \in \mathcal{F}_{t}, i \geq 1$. Then $\Gamma_{1}:=\widetilde{\Gamma}_{1}, \Gamma_{i}:=\widetilde{\Gamma}_{i} \backslash\left(\cup_{l=1}^{i-1} \widetilde{\Gamma}_{l}\right) \in \mathcal{F}_{t}, i \geq 2$, form an $\left(\Omega, \mathcal{F}_{t}\right)$-partition, and $u_{1}^{\varepsilon}:=\sum_{i \geq 1} \mathbf{1}_{\Gamma_{i}} u_{i}^{1}$ belongs obviously to $\mathcal{U}_{t, t+\delta}$. Moreover, from the nonanticipativity of $\beta_{1}$ we have $\beta_{1}\left(u_{1}^{\varepsilon}\right)=\sum_{i \geq 1} \mathbf{1}_{\Gamma_{i}} \beta_{1}\left(u_{i}^{1}\right)$, and from the uniqueness of the solution of $\operatorname{SDE}(3.1)$ and $\operatorname{RBSDE}(3.5)$, we deduce that $I_{\delta}\left(t, x, u_{1}^{\varepsilon}, \beta_{1}\left(u_{1}^{\varepsilon}\right)\right)=$ $\sum_{i \geq 1} \mathbf{1}_{\Gamma_{i}} I_{\delta}\left(t, x, u_{i}^{1}, \beta_{1}\left(u_{i}^{1}\right)\right)$, P-a.s. Hence,

$$
\begin{aligned}
W_{\delta}(t, x) \leq I_{\delta}\left(t, x, \beta_{1}\right) & \leq \sum_{i \geq 1} \mathbf{1}_{\Gamma_{i}} I_{\delta}\left(t, x, u_{i}^{1}, \beta_{1}\left(u_{i}^{1}\right)\right)+\varepsilon=I_{\delta}\left(t, x, u_{1}^{\varepsilon}, \beta_{1}\left(u_{1}^{\varepsilon}\right)\right)+\varepsilon \\
& =G_{t, t+\delta}^{t, x ; u_{1}^{\varepsilon}, \beta_{1}\left(u_{1}^{\varepsilon}\right)}\left[W\left(t+\delta, X_{t+\delta}^{t, x ; u_{1}^{\varepsilon}, \beta_{1}\left(u_{1}^{\varepsilon}\right)}\right)\right]+\varepsilon, \text { P-a.s. }
\end{aligned}
$$

On the other hand, using the fact that $\beta_{1}(\cdot):=\beta\left(\cdot \oplus u_{2}\right) \in \mathcal{B}_{t, t+\delta}$ does not depend on $u_{2}(\cdot) \in \mathcal{U}_{t+\delta, T}$ we can define $\beta_{2}\left(u_{2}\right):=\left.\beta\left(u_{1}^{\varepsilon} \oplus u_{2}\right)\right|_{[t+\delta, T]}$, for all $u_{2}(\cdot) \in \mathcal{U}_{t+\delta, T}$. The such defined $\beta_{2}: \mathcal{U}_{t+\delta, T} \rightarrow \mathcal{V}_{t+\delta, T}$ belongs to $\mathcal{B}_{t+\delta, T}$ since $\beta \in \mathcal{B}_{t, T}$. Therefore, from the definition of $W(t+\delta, y)$ we have, for any $y \in \mathbb{R}^{n}$,

$$
W(t+\delta, y) \leq \operatorname{esssup}_{u_{2} \in \mathcal{U}_{t+\delta, T}} J\left(t+\delta, y ; u_{2}, \beta_{2}\left(u_{2}\right)\right), \text { P-a.s. }
$$

Finally, because there exists a constant $C \in \mathbb{R}$ such that

(i) $\left|W(t+\delta, y)-W\left(t+\delta, y^{\prime}\right)\right| \leq C\left|y-y^{\prime}\right|$, for any $y, y^{\prime} \in \mathbb{R}^{n}$;

(ii) $\left|J\left(t+\delta, y, u_{2}, \beta_{2}\left(u_{2}\right)\right)-J\left(t+\delta, y^{\prime}, u_{2}, \beta_{2}\left(u_{2}\right)\right)\right| \leq C\left|y-y^{\prime}\right|$, P-a.s., for any $u_{2} \in \mathcal{U}_{t+\delta, T}$,

(see Lemma 3.2-(i) and (3.6)-(i)) we can show by approximating $X_{t+\delta}^{t, x ; u_{1}^{\varepsilon}, \beta_{1}\left(u_{1}^{\varepsilon}\right)}$ by finite-valued $\mathcal{F}_{t+\delta}$-measurable random vectors that

$W\left(t+\delta, X_{t+\delta}^{t, x ; u_{1}^{\varepsilon}, \beta_{1}\left(u_{1}^{\varepsilon}\right)}\right) \leq \operatorname{esssup}_{u_{2} \in \mathcal{U}_{t+\delta, T}} J\left(t+\delta, X_{t+\delta}^{t, x ; u_{1}^{\varepsilon}, \beta_{1}\left(u_{1}^{\varepsilon}\right)} ; u_{2}, \beta_{2}\left(u_{2}\right)\right)$, P-a.s.

To estimate the right side of the latter inequality we note that there exists some sequence $\left\{u_{j}^{2}, j \geq 1\right\} \subset \mathcal{U}_{t+\delta, T}$ such that

$$
\begin{aligned}
& \operatorname{esssup}_{u_{2} \in \mathcal{U}_{t+\delta, T}} J\left(t+\delta, X_{t+\delta}^{t, x ; u_{1}^{\varepsilon}, \beta_{1}\left(u_{1}^{\varepsilon}\right)} ; u_{2}, \beta_{2}\left(u_{2}\right)\right) \\
& =\sup _{j \geq 1} J\left(t+\delta, X_{t+\delta}^{t, x ; u_{1}^{\varepsilon}, \beta_{1}\left(u_{1}^{\varepsilon}\right)} ; u_{j}^{2}, \beta_{2}\left(u_{j}^{2}\right)\right) \text {, P-a.s. }
\end{aligned}
$$

Then, putting $\widetilde{\Delta}_{j}:=\left\{\operatorname{esssup}_{u_{2} \in \mathcal{U}_{t+\delta, T}} J\left(t+\delta, X_{t+\delta}^{t, x ; u_{1}^{\varepsilon}, \beta_{1}\left(u_{1}^{\varepsilon}\right)} ; u_{2}, \beta_{2}\left(u_{2}\right)\right) \leq J(t+\right.$ $\left.\left.\delta, X_{t+\delta}^{t, x ; u_{1}^{\varepsilon}, \beta_{1}\left(u_{1}^{\varepsilon}\right)} ; u_{j}^{2}, \beta_{2}\left(u_{j}^{2}\right)\right)+\varepsilon\right\} \in \mathcal{F}_{t+\delta}, j \geq 1$; we have with $\Delta_{1}:=\widetilde{\Delta}_{1}, \Delta_{j}:=$ $\widetilde{\Delta}_{j} \backslash\left(\cup_{l=1}^{j-1} \widetilde{\Delta}_{l}\right) \in \mathcal{F}_{t+\delta}, j \geq 2$, an $\left(\Omega, \mathcal{F}_{t+\delta}\right)$-partition and $u_{2}^{\varepsilon}:=\sum_{j \geq 1} \mathbf{1}_{\Delta_{j}} u_{j}^{2} \in$ $\mathcal{U}_{t+\delta, T}$. From the nonanticipativity of $\beta_{2}$ we have $\beta_{2}\left(u_{2}^{\varepsilon}\right)=\sum_{j \geq 1} \mathbf{1}_{\Delta_{j}} \bar{\beta}_{2}\left(u_{j}^{2}\right)$, and 
from the definition of $\beta_{1}$ and $\beta_{2}$ we know that $\beta\left(u_{1}^{\varepsilon} \oplus u_{2}^{\varepsilon}\right)=\beta_{1}\left(u_{1}^{\varepsilon}\right) \oplus \beta_{2}\left(u_{2}^{\varepsilon}\right)$. Thus, again from the uniqueness of the solution of our FBSDE, we get

$$
\begin{aligned}
& J\left(t+\delta, X_{t+\delta}^{t, x ; u_{1}^{\varepsilon}, \beta_{1}\left(u_{1}^{\varepsilon}\right)} ; u_{2}^{\varepsilon}, \beta_{2}\left(u_{2}^{\varepsilon}\right)\right) \\
& =Y_{t+\delta}^{t+\delta, X_{t+\delta}^{t, x ; u_{1}^{\varepsilon}, \beta_{1}\left(u_{1}^{\varepsilon}\right)} ; u_{2}^{\varepsilon}, \beta_{2}\left(u_{2}^{\varepsilon}\right)}
\end{aligned}
$$

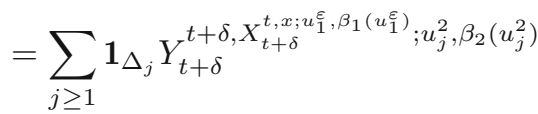

$$
\begin{aligned}
& =\sum_{j \geq 1} \mathbf{1}_{\Delta_{j}} J\left(t+\delta, X_{t+\delta}^{t, x ; u_{1}^{\varepsilon}, \beta_{1}\left(u_{1}^{\varepsilon}\right)} ; u_{j}^{2}, \beta_{2}\left(u_{j}^{2}\right)\right) \text {, P-a.s. }
\end{aligned}
$$

Consequently,

$$
\begin{aligned}
W\left(t+\delta, X_{t+\delta}^{t, x ; u_{1}^{\varepsilon}, \beta_{1}\left(u_{1}^{\varepsilon}\right)}\right) & \leq \operatorname{esssup}_{u_{2} \in \mathcal{U}_{t+\delta, T}} J\left(t+\delta, X_{t+\delta}^{t, x ; u_{1}^{\varepsilon}, \beta_{1}\left(u_{1}^{\varepsilon}\right)} ; u_{2}, \beta_{2}\left(u_{2}\right)\right) \\
& \leq J\left(t+\delta, X_{t+, i u_{1}^{\varepsilon}, \beta_{1}\left(u_{1}^{\varepsilon}\right)}^{t} ; u_{2}^{\varepsilon}, \beta_{2}\left(u_{2}^{\varepsilon}\right)\right)+\varepsilon \\
& =Y_{t+\delta}^{t+\delta, X_{t+\delta}^{t, x ; u_{1}^{\varepsilon}, \beta_{1}\left(u_{1}^{\varepsilon}\right)} ; u_{2}^{\varepsilon}, \beta_{2}\left(u_{2}^{\varepsilon}\right)}+\varepsilon \\
& =Y_{t+\delta ; u_{1}^{\varepsilon} \oplus u_{2}^{\varepsilon}, \beta\left(u_{1}^{\varepsilon} \oplus u_{2}^{\varepsilon}\right)}^{t, \delta}+\varepsilon \\
& =Y_{t+\delta}^{t, x ; u^{\varepsilon}, \beta\left(u^{\varepsilon}\right)}+\varepsilon, \text { P-a.s. }
\end{aligned}
$$

where $u^{\varepsilon}:=u_{1}^{\varepsilon} \oplus u_{2}^{\varepsilon} \in \mathcal{U}_{t, T}$. From (6.21) and the definition of $W$ we conclude:

$$
\begin{aligned}
& h\left(t+\delta, X_{t+\delta}^{t, x ; u^{\varepsilon}, \beta\left(u^{\varepsilon}\right)}\right) \leq W\left(t+\delta, X_{t+\delta}^{t, x ; u_{1}^{\varepsilon}, \beta_{1}\left(u_{1}^{\varepsilon}\right)}\right) \\
& \quad \leq\left(Y_{t+\delta}^{t, x ; u^{\varepsilon}, \beta\left(u^{\varepsilon}\right)}+\varepsilon\right) \wedge h^{\prime}\left(t+\delta, X_{t+\delta}^{t, x ; u^{\varepsilon}, \beta\left(u^{\varepsilon}\right)}\right),
\end{aligned}
$$

$P$-a.s. From (6.19), (6.21) and the comparison theorem for BSDEs with two reflecting boundaries we then get:

$$
W_{\delta}(t, x) \leq G_{t, t+\delta}^{t, x ; u_{1}^{\varepsilon}, \beta_{1}\left(u_{1}^{\varepsilon}\right)}\left[\left(Y_{t+\delta}^{t, x ; u^{\varepsilon}, \beta\left(u^{\varepsilon}\right)}+\varepsilon\right) \wedge h^{\prime}\left(t+\delta, X_{t+\delta}^{t, x ; u^{\varepsilon}, \beta\left(u^{\varepsilon}\right)}\right)\right]+\varepsilon .
$$

Thus, taking into account that $Y_{t+\delta}^{t, x ; u^{\varepsilon}, \beta\left(u^{\varepsilon}\right)} \leq h^{\prime}\left(t+\delta, X_{t+\delta}^{t, x ; u^{\varepsilon}, \beta\left(u^{\varepsilon}\right)}\right)$, we deduce from Lemma 2.9:

$$
\begin{aligned}
W_{\delta}(t, x) & \leq G_{t, t+\delta}^{t, x ; u_{1}^{\varepsilon}, \beta_{1}\left(u_{1}^{\varepsilon}\right)}\left[\left(Y_{t+\delta}^{t, x ; u^{\varepsilon}, \beta\left(u^{\varepsilon}\right)}+\varepsilon\right) \wedge h^{\prime}\left(t+\delta, X_{t+\delta}^{t, x ; u^{\varepsilon}, \beta\left(u^{\varepsilon}\right)}\right)\right]+\varepsilon \\
& \leq G_{t, t+\delta}^{t, x ; u_{1}^{\varepsilon}, \beta_{1}\left(u_{1}^{\varepsilon}\right)}\left[Y_{t+\delta}^{t, x ; u^{\varepsilon}, \beta\left(u^{\varepsilon}\right)}\right]+(C+1) \varepsilon \\
& =G_{t, t+\delta}^{t, x ; u^{\varepsilon}, \beta\left(u^{\varepsilon}\right)}\left[Y_{t+\delta}^{t, x ; u^{\varepsilon}, \beta\left(u^{\varepsilon}\right)}\right]+(C+1) \varepsilon \\
& =Y_{t}^{t, x ; u^{\varepsilon}, \beta\left(u^{\varepsilon}\right)}+(C+1) \varepsilon \\
& \leq \operatorname{esssup}_{u \in \mathcal{U}_{t, T}} Y_{t}^{t, x ; u, \beta(u)}+(C+1) \varepsilon, \text { P-a.s. }
\end{aligned}
$$

Therefore, in virtue of the arbitrariness of $\beta \in \mathcal{B}_{t, T}$,

$$
W_{\delta}(t, x) \leq \operatorname{essinf}_{\beta \in \mathcal{B}_{t, T}} \operatorname{esssup}_{u \in \mathcal{U}_{t, T}} Y_{t}^{t, x ; u, \beta(u)}+(C+1) \varepsilon=W(t, x)+(C+1) \varepsilon .
$$

Finally, letting $\varepsilon \downarrow 0$, we get $W_{\delta}(t, x) \leq W(t, x)$. 
Lemma 6.3. $W(t, x) \leq W_{\delta}(t, x)$.

Proof. We continue to use the notations introduced above. From the definition of $W_{\delta}(t, x)$ we have

$$
\begin{aligned}
W_{\delta}(t, x) & =\operatorname{essinf}_{\beta_{1} \in \mathcal{B}_{t, t+\delta}} \operatorname{esssup}_{u_{1} \in \mathcal{U}_{t, t+\delta}} G_{t, t+\delta}^{t, x ; u_{1}, \beta_{1}\left(u_{1}\right)}\left[W\left(t+\delta, X_{t+\delta}^{t, x ; u_{1}, \beta_{1}\left(u_{1}\right)}\right)\right] \\
& =\operatorname{essinf}_{\beta_{1} \in \mathcal{B}_{t, t+\delta}} I_{\delta}\left(t, x, \beta_{1}\right),
\end{aligned}
$$

where we have put

$$
I_{\delta}\left(t, x, \beta_{1}\right):=\operatorname{esssup}_{u_{1} \in \mathcal{U}_{t, t+\delta}} I_{\delta}\left(t, x, u_{1}, \beta_{1}\left(u_{1}\right)\right) .
$$

We select $\left\{\beta_{i}^{1}, i \geq 1\right\} \subset \mathcal{B}_{t, t+\delta}$ such that

$$
W_{\delta}(t, x)=\inf _{i \geq 1} I_{\delta}\left(t, x, \beta_{i}^{1}\right), \text { P-a.s. }
$$

and for an arbitrarily small $\varepsilon>0$ we put $\widetilde{\Lambda}_{i}:=\left\{I_{\delta}\left(t, x, \beta_{i}^{1}\right)-\varepsilon \leq W_{\delta}(t, x)\right\} \in$ $\mathcal{F}_{t}, \quad i \geq 1, \Lambda_{1}:=\widetilde{\Lambda}_{1}$ and $\Lambda_{i}:=\widetilde{\Lambda}_{i} \backslash\left(\cup_{l=1}^{i-1} \widetilde{\Lambda}_{l}\right) \in \mathcal{F}_{t}, i \geq 2$. Then $\left\{\Lambda_{i}, \quad i \geq\right.$ $1\}$ is an $\left(\Omega, \mathcal{F}_{t}\right)$-partition, $\beta_{1}^{\varepsilon}:=\sum_{i \geq 1} \mathbf{1}_{\Lambda_{i}} \beta_{i}^{1}$ belongs to $\mathcal{B}_{t, t+\delta}$, and from the uniqueness of the solution of our FBSDE we conclude that $I_{\delta}\left(t, x, u_{1}, \beta_{1}^{\varepsilon}\left(u_{1}\right)\right)=$ $\sum_{i \geq 1} \mathbf{1}_{\Lambda_{i}} I_{\delta}\left(t, x, u_{1}, \beta_{i}^{1}\left(u_{1}\right)\right)$, P-a.s., for all $u_{1}(\cdot) \in \mathcal{U}_{t, t+\delta}$. Hence,

$$
\begin{aligned}
W_{\delta}(t, x) \geq & \sum_{i \geq 1} \mathbf{1}_{\Lambda_{i}} I_{\delta}\left(t, x, \beta_{i}^{1}\right)-\varepsilon \\
\geq & \sum_{i \geq 1} \mathbf{1}_{\Lambda_{i}} I_{\delta}\left(t, x, u_{1}, \beta_{i}^{1}\left(u_{1}\right)\right)-\varepsilon \\
= & I_{\delta}\left(t, x, u_{1}, \beta_{1}^{\varepsilon}\left(u_{1}\right)\right)-\varepsilon \\
= & G_{t, t+\delta}^{t, x}, \beta_{1}^{\varepsilon}\left(u_{1}\right)\left[W\left(t+\delta, X_{t+\delta}^{t, x ; u_{1}, \beta_{1}^{\varepsilon}\left(u_{1}\right)}\right)\right]-\varepsilon, \\
& \text { P-a.s., for all } u_{1} \in \mathcal{U}_{t, t+\delta} .
\end{aligned}
$$

On the other hand, from the definition of $W(t+\delta, y)$, with the same technique as before, we deduce that, for any $y \in \mathbb{R}^{n}$, there exists $\beta_{y}^{\varepsilon} \in \mathcal{B}_{t+\delta, T}$ such that

$$
W(t+\delta, y) \geq \operatorname{esssup}_{u_{2} \in \mathcal{U}_{t+\delta, T}} J\left(t+\delta, y ; u_{2}, \beta_{y}^{\varepsilon}\left(u_{2}\right)\right)-\varepsilon, \text { P-a.s. }
$$

For every $m \geq 1$ we now introduce

$$
\varepsilon_{m}:=\left(\frac{1}{3} \inf _{m-1 \leq|x| \leq m}\left(h^{\prime}(t+\delta, x)-h(t+\delta, x)\right)\right) \wedge \varepsilon>0 .
$$

Letting $C_{0}>0$ be the common Lipschitz constant of $h^{\prime}(t+\delta,$.$) and h(t+\delta,$. we put $\delta_{m}=C_{0}^{-1} \varepsilon_{m}$. Moreover, we let $\mathcal{O}_{i}^{m}, i \geq 1$, be a Borel measurable decomposition of $\Lambda_{m}:=\left\{x \in R^{n}: m-1 \leq|x|<m\right\}$ such that $\sum_{i>1} \mathcal{O}_{i}^{m}=\Lambda_{m}$ and $\operatorname{diam}\left(\mathcal{O}_{i}^{m}\right) \leq \delta_{m}, i \geq 1$. For each $m, i \geq 1$ we choose an arbitrary element of $y_{i}^{m} \in \mathcal{O}_{i}^{m}$. Then, defining $\left[X_{t+\delta}^{t, x ; u_{1}, \beta_{1}^{\varepsilon}\left(u_{1}\right)}\right]:=\sum_{i, m \geq 1} y_{i}^{m} \mathbf{1}_{\left\{X_{t+\delta}^{t, x ; u_{1}, \beta_{1}^{\varepsilon}\left(u_{1}\right)} \in \mathcal{O}_{i}^{m}\right\}}$, we have

$$
\left|X_{t+\delta}^{t, x ; u_{1}, \beta_{1}^{\varepsilon}\left(u_{1}\right)}-\left[X_{t+\delta}^{t, x ; u_{1}, \beta_{1}^{\varepsilon}\left(u_{1}\right)}\right]\right| \leq \delta_{m}
$$

and

$$
\left|h\left(t+\delta, X_{t+\delta}^{t, x ; u_{1}, \beta_{1}^{\varepsilon}\left(u_{1}\right)}\right)-h\left(t+\delta,\left[X_{t+\delta}^{t, x ; u_{1}, \beta_{1}^{\varepsilon}\left(u_{1}\right)}\right]\right)\right| \leq \varepsilon_{m},
$$


everywhere on $\left\{X_{t+\delta}^{t, x ; u_{1}, \beta_{1}^{\varepsilon}\left(u_{1}\right)} \in \Lambda_{m}\right\}$, for all $u_{1} \in \mathcal{U}_{t, t+\delta}$. The same result also holds for $h^{\prime}$ at the place of $h$. Then,

$$
\begin{aligned}
h\left(t+\delta, X_{t+\delta}^{t, x ; u_{1}, \beta_{1}^{\varepsilon}\left(u_{1}\right)}\right) & \leq h\left(t+\delta,\left[X_{t+\delta}^{t, x ; u_{1}, \beta_{1}^{\varepsilon}\left(u_{1}\right)}\right]\right)+\varepsilon_{m} \\
& <h^{\prime}\left(t+\delta,\left[X_{t+\delta}^{t, x ; u_{1}, \beta_{1}^{\varepsilon}\left(u_{1}\right)}\right]\right)-\varepsilon_{m} \\
& \leq h^{\prime}\left(t+\delta, X_{t+\delta}^{t, x ; u_{1}, \beta_{1}^{\varepsilon}\left(u_{1}\right)}\right)
\end{aligned}
$$

on $\left\{X_{t+\delta}^{t, x ; u_{1}, \beta_{1}^{\varepsilon}\left(u_{1}\right)} \in \Lambda_{m}\right\}$, for all $u_{1} \in \mathcal{U}_{t, t+\delta}$. We choose for every $y_{i}^{m}$ some $\beta_{y_{i}^{m}}^{\varepsilon} \in$ $\mathcal{B}_{t+\delta, T}$ such that (6.24) is fulfilled, and clearly $\beta_{u_{1}}^{\varepsilon}:=\sum_{i, m \geq 1} \mathbf{1}_{\left\{X_{t+\delta}^{t, x ; u_{1}, \beta_{1}^{\varepsilon}\left(u_{1}\right)} \in \mathcal{O}_{i}^{m}\right\}}$ $\beta_{y_{i}^{m}}^{\varepsilon} \in \mathcal{B}_{t+\delta, T}$.

Now we can define the new strategy $\beta^{\varepsilon}(u):=\beta_{1}^{\varepsilon}\left(u_{1}\right) \oplus \beta_{u_{1}}^{\varepsilon}\left(u_{2}\right), u \in \mathcal{U}_{t, T}$, where $u_{1}=\left.u\right|_{[t, t+\delta]}, u_{2}=\left.u\right|_{(t+\delta, T]}$ (restriction of $u$ to $[t, t+\delta] \times \Omega$ and $(t+\delta, T] \times \Omega$, resp.). Obviously, $\beta^{\varepsilon}$ maps $\mathcal{U}_{t, T}$ into $\mathcal{V}_{t, T}$. Moreover, $\beta^{\varepsilon}$ is nonanticipating: Indeed, let $S: \Omega \longrightarrow[t, T]$ be an $\mathbb{F}$-stopping time and $u, u^{\prime} \in \mathcal{U}_{t, T}$ be such that $u \equiv u^{\prime}$ on $\llbracket t, S \rrbracket$. Decomposing $u, u^{\prime}$ into $u_{1}, u_{1}^{\prime} \in \mathcal{U}_{t, t+\delta}, u_{2}, u_{2}^{\prime} \in \mathcal{U}_{t+\delta, T}$ such that $u=u_{1} \oplus u_{2}$ and $u^{\prime}=u_{1}^{\prime} \oplus u_{2}^{\prime}$ we have $u_{1} \equiv u_{1}^{\prime}$ on $\llbracket t, S \wedge(t+\delta) \rrbracket$, from where we get $\beta_{1}^{\varepsilon}\left(u_{1}\right) \equiv \beta_{1}^{\varepsilon}\left(u_{1}^{\prime}\right)$ on $\llbracket t, S \wedge(t+\delta) \rrbracket$ (recall that $\beta_{1}^{\varepsilon}$ is nonanticipating). On the other hand, $u_{2} \equiv u_{2}^{\prime}$ on $\rrbracket t+\delta, S \vee(t+\delta) \rrbracket(\subset(t+\delta, T] \times\{S>t+\delta\})$, and on $\{S>t+\delta\}$ we have $X_{t+\delta}^{t, x ; u_{1}, \beta_{1}^{\varepsilon}\left(u_{1}\right)}=X_{t+\delta}^{t, x ; u_{1}^{\prime}, \beta_{1}^{\varepsilon}\left(u_{1}^{\prime}\right)}$. Consequently, from our definition, $\beta_{u_{1}}^{\varepsilon}=\beta_{u_{1}^{\prime}}^{\varepsilon}$ on $\{S>t+\delta\}$ and $\beta_{u_{1}}^{\varepsilon}\left(u_{2}\right) \equiv \beta_{u_{1}^{\prime}}^{\varepsilon}\left(u_{2}^{\prime}\right)$ on $\rrbracket t+\delta, S \vee(t+\delta) \rrbracket$. This yields $\beta^{\varepsilon}(u)=\beta_{1}^{\varepsilon}\left(u_{1}\right) \oplus \beta_{u_{1}}^{\varepsilon}\left(u_{2}\right) \equiv \beta_{1}^{\varepsilon}\left(u_{1}^{\prime}\right) \oplus \beta_{u_{1}^{\prime}}^{\varepsilon}\left(u_{2}^{\prime}\right)=\beta^{\varepsilon}\left(u^{\prime}\right)$ on $\llbracket t, S \rrbracket$, from where it follows that $\beta^{\varepsilon} \in \mathcal{B}_{t, T}$.

Let now $u \in \mathcal{U}_{t, T}$ be arbitrarily chosen and decomposed into $u_{1}=\left.u\right|_{[t, t+\delta]} \in$ $\mathcal{U}_{t, t+\delta}$ and $u_{2}=\left.u\right|_{(t+\delta, T]} \in \mathcal{U}_{t+\delta, T}$. Then, with the notations

$$
\begin{gathered}
W_{\varepsilon}(t+\delta, x):=\max \left\{h(t+\delta, x)+\varepsilon_{m}, \min \left\{W(t+\delta, x), h^{\prime}(t+\delta, x)-\varepsilon_{m}\right\}\right\}, \\
J_{\varepsilon}(t+\delta, x ; u, v):=\max \left\{h(t+\delta, x)+\varepsilon_{m}, \min \left\{J(t+\delta, x ; u, v), h^{\prime}(t+\delta, x)-\varepsilon_{m}\right\}\right\} \text { and } \\
\widehat{J}_{\varepsilon}(t+\delta, x ; u, v):=\max \left\{h(t+\delta, x)+\varepsilon_{m}, \min \left\{J(t+\delta, x ; u, v)-\varepsilon, h^{\prime}(t+\delta, x)-\varepsilon_{m}\right\}\right\} \\
=\max \left\{h(t+\delta, x)+\varepsilon_{m}, J(t+\delta, x ; u, v)-\varepsilon\right\},
\end{gathered}
$$

for $x \in \Lambda_{m}, m \geq 1, u \in \mathcal{U}_{t+\delta, T}$ and $v \in \mathcal{V}_{t+\delta, T}$, we have obviously,

$$
\begin{gathered}
\widehat{J}_{\varepsilon}\left(t+\delta,\left[X_{t+\delta}^{t, x ; u_{1}, \beta_{1}^{\varepsilon}\left(u_{1}\right)}\right] ; u, v\right), J_{\varepsilon}\left(t+\delta,\left[X_{t+\delta}^{t, x ; u_{1}, \beta_{1}^{\varepsilon}\left(u_{1}\right)}\right] ; u, v\right), \\
W_{\varepsilon}\left(t+\delta,\left[X_{t+\delta}^{t, x ; u_{1}, \beta_{1}^{\varepsilon}\left(u_{1}\right)}\right] ; u, v\right) \in\left[h\left(t+\delta, X_{t+\delta}^{t, x ; u_{1}, \beta_{1}^{\varepsilon}\left(u_{1}\right)}\right),\right. \\
\left.h^{\prime}\left(t+\delta, X_{t+\delta}^{t, x ; u_{1}, \beta_{1}^{\varepsilon}\left(u_{1}\right)}\right)\right],
\end{gathered}
$$

and

$$
\begin{gathered}
\left|J_{\varepsilon}\left(t+\delta,\left[X_{t+\delta}^{t, x ; u_{1}, \beta_{1}^{\varepsilon}\left(u_{1}\right)}\right] ; u, v\right)-J\left(t+\delta, X_{t+\delta}^{t, x ; u_{1}, \beta_{1}^{\varepsilon}\left(u_{1}\right)} ; u, v\right)\right| \leq C \varepsilon, \\
\left|W_{\varepsilon}\left(t+\delta,\left[X_{t+\delta}^{t, x ; u_{1}, \beta_{1}^{\varepsilon}\left(u_{1}\right)}\right]\right)-W\left(t+\delta, X_{t+\delta}^{t, x ; u_{1}, \beta_{1}^{\varepsilon}\left(u_{1}\right)}\right)\right| \leq C \varepsilon,
\end{gathered}
$$


where $C$ is a constant which is independent of $\varepsilon$ and the control processes. Thus, from (6.23),

$$
\begin{aligned}
W_{\delta}(t, x) & \geq G_{t, t+\delta}^{t, x ; u_{1}, \beta_{1}^{\varepsilon}\left(u_{1}\right)}\left[W\left(t+\delta, X_{t+\delta}^{t, x ; u_{1}, \beta_{1}^{\varepsilon}\left(u_{1}\right)}\right)\right]-\varepsilon \\
& \geq G_{t, t+\delta}^{t, x ; u_{1}, \beta_{1}^{\varepsilon}\left(u_{1}\right)}\left[W_{\varepsilon}\left(t+\delta,\left[X_{t+\delta}^{t, x ; u_{1}, \beta_{1}^{\varepsilon}\left(u_{1}\right)}\right]\right)\right]-C \varepsilon \\
& =G_{t, t+\delta}^{t, x ; u_{1}, \beta_{1}^{\varepsilon}\left(u_{1}\right)}\left[\sum_{i, m \geq 1} \mathbf{1}_{\left\{X_{t+\delta}^{t, x ; u_{1}, \beta_{1}^{\varepsilon}\left(u_{1}\right)} \in \mathcal{O}_{i}^{m}\right\}} W_{\varepsilon}\left(t+\delta, y_{i}^{m}\right)\right]-C \varepsilon
\end{aligned}
$$

and, since

$\left|\widehat{J}_{\varepsilon}(t+\delta, y ; u, v)-J_{\varepsilon}(t+\delta, y ; u, v)\right| \leq \varepsilon$, for all $y \in \mathbb{R}^{n}, u \in \mathcal{U}_{t+\delta, T}, v \in \mathcal{V}_{t+\delta, T}$,

we obtain

$$
\begin{aligned}
W_{\delta}(t, x) & \geq G_{t, t+\delta}^{t, x ; u_{1}, \beta_{1}^{\varepsilon}\left(u_{1}\right)}\left[\sum_{i, m \geq 1} \mathbf{1}_{\left\{X_{t+\delta}^{\left.t, x ; u_{1}, \beta_{1}^{\varepsilon}\left(u_{1}\right) \in \mathcal{O}_{i}^{m}\right\}}\right.} \widehat{J}_{\varepsilon}\left(t+\delta, y_{i}^{m} ; u_{2}, \beta_{y_{i}^{m}}^{\varepsilon}\left(u_{2}\right)\right)\right]-C \varepsilon \\
& \geq G_{t, t+\delta}^{t, x ; u_{1}, \beta_{1}^{\varepsilon}\left(u_{1}\right)}\left[\sum_{i, m \geq 1} \mathbf{1}_{\left\{X_{t+\delta}^{t, x ; u_{1}, \beta_{1}^{\varepsilon}\left(u_{1}\right)} \in \mathcal{O}_{i}^{m}\right\}} J_{\varepsilon}\left(t+\delta, y_{i}^{m} ; u_{2}, \beta_{y_{i}^{m}}^{\varepsilon}\left(u_{2}\right)\right)\right]-C \varepsilon \\
& =G_{t, t+\delta}^{t, x ; u_{1}, \beta_{1}^{\varepsilon}\left(u_{1}\right)}\left[J_{\varepsilon}\left(t+\delta,\left[X_{t+\delta}^{t, x ; u_{1}, \beta_{1}^{\varepsilon}\left(u_{1}\right)}\right] ; u_{2}, \beta_{u_{1}}^{\varepsilon}\left(u_{2}\right)\right)\right]-C \varepsilon \\
& \geq G_{t, t+\delta}^{t, x ; u_{1}, \beta_{1}^{\varepsilon}\left(u_{1}\right)}\left[J\left(t+\delta, X_{t+\delta}^{t, x ; u_{1}, \beta_{1}^{\varepsilon}\left(u_{1}\right)} ; u_{2}, \beta_{u_{1}}^{\varepsilon}\left(u_{2}\right)\right)\right]-C \varepsilon \\
& =G_{t, t+\delta}^{t, x ; u, \beta^{\varepsilon}(u)}\left[Y_{t+\delta}^{t, x ; u, \beta^{\varepsilon}(u)}\right]-C \varepsilon \\
& =Y_{t}^{t, x ; u, \beta^{\varepsilon}(u)}-C \varepsilon, P \text {-a.s., for any } u \in \mathcal{U}_{t, T} .
\end{aligned}
$$

(Notice that the constant $C$ may be different from line to line). This allows to conclude that $W_{\delta}(t, x) \geq W(t, x)-C \varepsilon$, and we get the wished relation by letting $\varepsilon \rightarrow 0$.

\section{Acknowledgment}

Both authors thank the referee for his helpful suggestions. Juan Li thanks Mingyu $\mathrm{Xu}$ for some helpful discussions on RBSDEs with two barriers.

\section{References}

[1] Barles, G., Buckdahn, R., Pardoux, E.: Backward stochastic differential equations and integral-partial differential equations. Stoch. Stoch. Rep. 60, 57-83 (1997)

[2] Buckdahn, R., Cardaliaguet, P., Rainer, C.: Nash equilibrium payoffs for nonzerosum stochastic differential games. SIAM J. Cont. Opt. 43(2), 624-642 (2004) 
[3] Buckdahn, R., LI, J.: Stochastic differential games and viscosity solutions of Hamilton-Jacobi-Bellman-Isaacs equations. SIAM J. Cont. Opt. 47(1), 444-475 (2008). doi: 10.1137/060671954

[4] Buckdahn, R., LI, J.: Stochastic differential games with reflection and related obstacle problems for Isaacs equations. Submitted. (2007) Available online: http://arxiv. org/abs/0707.1133

[5] Crandall, M.G., Ishii, H., Lions, P.L.: User's guide to viscosity solutions of second order partial differential equations. Bull. Am. Math. Soc. 27, 1-67 (1992)

[6] Cvitanic, J., Karatzas, I.: Backward SDE's with reflection and Dynkin Games. Ann. Probab. 24, 2024-2056 (1996)

[7] Dellacherie, C.: Sur l'existence de certains essinf et esssup de familles de processus mesuables. In: Sem. Probab. XII. Lecture Notes in Math. vol. 649, pp. 512-514. Springer-Verlag, Berlin (1977)

[8] Dunford, N., Schwartz, J.T.: Linear Operators. Part I: General Theory. WileyInterscience, New York (1957)

[9] El Karoui, N., Peng, S., Quenez, M.C.: Backward stochastic differential equations in finance. Math. Finance. 7(1), 1-71 (1997)

[10] El Karoui, N., Kapoudjian, C., Pardoux, E., Peng, S., Quenez, M.C.: Reflected solutions of backward SDE's, and related obstacle problems for PDE's. Ann. Probab 25(2), 702-737 (1997)

[11] El Karoui, N., Pardoux, E., Quenez, M.C.: Reflected backward SDEs and American options. In: Numerical methods in finance. Publ. Newton Inst. pp. 215-231. Cambridge University Press, Cambridge (1997)

[12] Fleming, W.H., Souganidis, P.E.: On the existence of value functions of two-player, zero-sum stochastic differential games. Indiana Univ. Math. J. 38(2), 293-314 (1989)

[13] Hamadène, S.: Mixed zero-sum stochastic differential game and American game option. SIAM J. Con. Opt. 45, 496-518 (2006)

[14] Hamadène, S., Lepeltier, J.P.: Zero-sum stochastic differential games and backward equations. Syst. Control Lett. 24, 259-263 (1995)

[15] Hamadène, S., Lepeltier, J.P.: Reflected BSDEs and mixed game problems. Stoch. Process. Appl. 85, 177-188 (2000)

[16] Hamadène, S., Lepeltier, J.P., Matoussi, A.: Double barrier reflected backward stochastic differential equations with continuous coefficient. Pitman Res. Notes. Math. Ser. 364, 115-128 (1997)

[17] Hamadène, S., Lepeltier, J.P., Peng, S.: BSDEs with continuous coefficients and stochastic differential games. In: El Karoui, N., Mazliak, L. (eds.) Backward Stochastic Differential Equations. Pitman Res. Notes Math. Ser., vol. 364, pp. 115-128. Longman, Harlow (1997) 
[18] Hamadène, S., Hassani, M.: BSDEs with two reflecting barriers: the general result. Probab. Theory Relat. Fields 132, 237-264 (2005)

[19] Hou, S., Tang, S.: Switching games of stochastic differential systems. SIAM J. Cont. Opt. 46(3), 900-929 (2007)

[20] Ikeda, N., Watanabe, S.: Stochastic Differential Equations and Diffusion Processes. North Holland-Kodansha, Amsterdam-Tokyo (1989)

[21] Karatzas, I., Shreve, S.E.: Brownian Motion and Stochastic Calculus. SpringerVerlag, New York (1987)

[22] Karatzas, I., Shreve, S.E.: Methods of Mathematical Finance. Springer, New York (1998)

[23] Ma, J., Cvitanic, J.: Reflected forward-backward SDEs and obstacle problems with boundary conditions. J. Appl. Math. Stoch. Anal. 14(2), 113-138 (2001)

[24] Pardoux, E., Peng, S.: Adapted solution of a backward stochastic differential equation. Syst. Control Lett. 14(1-2), 61-74 (1990)

[25] Peng, S.: BSDE and stochastic optimizations. In: Yan, J., Peng, S., Fang, S., Wu, L. (eds.) Topics in Stochastic Analysis, Ch.2. Science Press. Beijing (in Chinese) (1997)

[26] Peng, S.: A generalized dynamic programming principle and Hamilton-JacobiBellman equation. Stoch. Stoch. Reports. 38, 119-134 (1992)

[27] Peng, S., Xu, M.: The smallest $g$-supermartingale and refelcted BSDE with single and double $L^{2}$ obstacles. Ann. I. H. Poincare PR. 41, 605-630 (2005)

R. Buckdahn

Département de Mathématiques

Université de Bretagne Occidentale

6, avenue Victor-le-Gorgeu, CS 93837

29238 Brest Cedex 3, France

e-mail: Rainer.Buckdahn@univ-brest.fr

J. Li

School of Mathematics and Statistics

Shandong University at Weihai

264209 Weihai, People's Republic of China

e-mail: juanli@sdu.edu.cn

Received: 02 April 2008

Revised: 02 January 2009

Accepted: 03 February 2009 